\title{
Optical morphology of distant RATAN-600 radio galaxies from subarcsecond resolution NOT images ${ }^{\star}$
}

\author{
T. Pursimo ${ }^{1}$, K. Nilsson ${ }^{1}$, P. Teerikorpi ${ }^{1}$, A. Kopylov ${ }^{2}$, N. Soboleva ${ }^{3}$, Yu. Parijskij ${ }^{2}$, Yu. Baryshev ${ }^{4}$, O. Verkhodanov $^{2}$ \\ A. Temirova ${ }^{3}$, O. Zhelenkova $^{2}$, W. Goss ${ }^{5}$, A. Sillanpää ${ }^{1}$, and L.O. Takalo ${ }^{1}$ \\ 1 Tuorla Observatory, FIN-21500 Piikkiö, Finland \\ 2 Special Astrophysical Observatory, Radio Astronomy Sector, 35147 Niznij Arkhyz, Karachey-Cherkessia, Russia \\ 3 St. Petersburg Branch of the SAO, Radio Astronomy Laboratory, Pulkovskoe Shosse 65, 196140 St. Petersburg, Russia \\ 4 Astronomical Institute of St. Petersburg University, 198904 St. Petersburg, Russia \\ ${ }^{5}$ National Radio Astronomical observatory, P.O. Box 0, Socorro, New Mexico 87801, U.S.A.
}

Received February 27; accepted September 7, 1998

\begin{abstract}
We present direct imaging data of 22 ultra steep spectrum radio sources obtained at (or near) a subarcsecond seeing. The basic sample of 40 double radio sources was selected from the RATAN-600 catalogue. The FRII-structure has been confirmed with VLA and preliminary optical identifications which come from the 6 m-telescope. As the RATAN-600 flux limit at $3.9 \mathrm{GHz}$ $(\approx 10 \mathrm{mJy})$ is fainter than that of major surveys, the sample may have high- $z$ contents. This is also suggested by the faint magnitudes in the Hubble diagram. The final aim is to create a homogeneous sample of high- $z$ radio galaxies in a well defined strip around the sky, with faint radio limit and subarcsecond morphology down to $m_{R}=24$.

We could confirm 16 identifications down to $m_{R} \sim 24$. Most of the extended objects have multicomponent structures as expected from other surveys of high-redshift radio galaxies. We found five unresolved objects even with a subarcsecond seeing. Of the remaining six objects, three are extremely faint and the other three have such a complex environment that further observations are needed to confirm the optical identification.
\end{abstract}

Key words: galaxies: active; structure — radio continuum: galaxies

Send offprint requests to: T. Pursimo

* Based on observations made with the Nordic Optical Telescope, operated on the island of La Palma jointly by Denmark, Finland, Iceland, Norway and Sweden, in the Spanish Observatorio del Roque de los Muchacos of the Instituto de Astrophysica de Canarias.

\section{Introduction}

There are several reasons to increase the still rather meagre data on very high- $z$, powerful radio galaxies (e.g. McCarthy 1993; Pariskij et al. 1997). High- $z$ radio galaxies are unique laboratories for investigating the early stages of galaxy and AGN evolution at look-back times corresponding to more than $90 \%$ of the age of the universe derived from Friedmann models. They may be used as tracers of the first generation of galaxy clusters (Peacock \& Nicholson 1991; Peacock 1997) and of the physical state of the intergalactic space (Parijskij et al. 1996a). By highresolution one may study important morphological features related to e.g. merging activity and "star burst" regions.

At redshift $z>2,120$ radio galaxies are known at present (de Brueck et al. 1997), in comparison with about 250 radio loud quasars $\left(S_{5 \mathrm{GHz}}>0.03 \mathrm{Jy}\right.$ in Veron-Cetty \& Veron 1996), though the former are intrinsically more abundant. According to the popular unified scheme, both classes are the same thing. One can study the host galaxies and close environments of radio galaxies, but this is difficult for QSOs at a similar redshift.

One aspect, where even a single galaxy may be decisive, is the question of how close in time to the cosmological singularity it is possible to find galaxies, with normal stellar population and supermassive compact objects in their nuclei. Though the use of high- $z$ objects in classical cosmological tests is hampered by severe problems, development of such tests is still one aim of observational cosmology. To identify selection effects and evolution, large samples are required. One must increase identifications of very remote galaxies, also in view of the new generation ground and space telescopes, which will allow their study at high resolution. 


\subsection{Extension of identified USS sources to fainter fluxes}

It has been known since the late 70's (Tielens et al. 1979; Blumenthal \& Miley 1979) that radio sources with steep spectra are optically fainter (and hence probably more distant) than sources with flatter spectra. Later it was established that observing faint radio sources with ultra-steep spectra (USS) is an efficient way to detect radio galaxies at high redshifts (see e.g. McCarthy 1993). As the USS Fanaroff-Riley type II (FRII-type; Fanaroff \& Riley 1974) radio galaxies are not good "standard radio candles" and as the reason for the success of the spectrum criterium is not known (see e.g. Röttgering et al. 1994), it is not clear what the outcome will be when USS samples are extended to a progressively fainter flux limit. Fainter flux may imply 1) larger redshifts, 2) similar redshifts, though weaker radio luminosity, or 3 ) smaller redshifts and still weaker luminosities. The first alternative is most interesting, though cases 2 and 3 are also important: extension of the luminosity range will help one to uncover the influence of radio luminosity on the classical cosmological tests (angular size-redshift; Nilsson et al. 1993 and Hubble diagram; Eales et al. 1997) and to decide whether alignment effect depends primarily on redshift or luminosity.

The flux range where differential normalized source counts show steepening is generally regarded as the most promising hunting place for high redshift objects. Parijskij et al. (1991) pointed out that the bulk of the RATAN-600 sample (see below) has fluxes in the range of $10-50 \mathrm{mJy}$ at $3.9 \mathrm{GHz}$ where the normalized counts show a maximum steepening, usually interpreted as a cosmological effect. A similar steepening in the counts is seen separately for steep spectrum sources (Fig. 6 in Kellermann \& Wall 1987). It has been suggested (e.g. Röttgering et al. 1994) that the most effective way to find distant galaxies would be a USS sample with $S_{408} \sim 0.2-1$ Jy. Indeed, this has proven to be so since about $50 \%$ of the Röttgering et al. (1994) USS objects have $z>2$ (van Ojik et al. 1997). The bright end of the USS sources is well studied (e.g. 4C/USS, B2/1 Jy, MRC/1 Jy McCarthy (1993) and references therein) and recently fainter flux limits have been reached (e.g. B3/VLA $S_{408}>0.8$ Jy Thompson et al. 1994; ESO/Key-Project $S_{365}>0.3$ Jy Röttgering et al. 1994). However, in the Röttgering et al. (1994) sample $365 \mathrm{MHz}$ flux density distribution peaks at about $1 \mathrm{Jy}$.

\subsection{RATAN-600 (RC) and UTRAO catalogues}

This paper is part of a programme initiated at the Special Astrophysical Observatory (Russia) with the aim of searching distant radio galaxies and investigating the early evolutionary stages of the universe (Goss et al. 1992). We wish to extend the steep-spectrum criteria to fainter fluxes than previously. This is accomplished by RC and UTRAO catalogues (see Fig. 1).

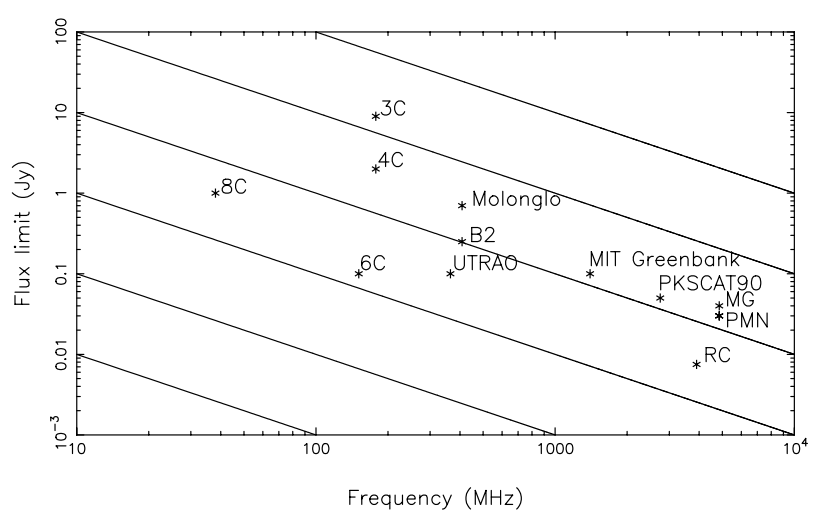

Fig. 1. Frequency - flux limit diagram with the positions of the RC sample and some other major radio catalogues. UTRAO $\left(-36^{\circ}<\delta<72^{\circ}\right)$ is the optimum low frequency catalogue presently available, which can be used for calculating the spectral index for a large part of the RC sample $\left(\delta \sim 5^{\circ}\right)$. Note that the $6 \mathrm{C}$ sample has $\delta>20^{\circ}$. The lines correspond to a source with $\alpha=1$

Our high frequency catalogue is based on a sample of faint radio sources originally discovered using the RATAN600 radio telescope in the "Kholod" ("Cold") experiment in 1980-81 (Parijskij et al. 1991; Parijskij et al. 1992; Parijskij \& Korolkov 1986). In the experiment, performed at $7.6 \mathrm{~cm}(3.9 \mathrm{GHz})$, the strip around the sky at $\delta=$ $5^{\circ} \pm 20^{\prime}$ was surveyed with a limiting flux of about $4 \mathrm{mJy}$. The RC catalogue resulted in containing 1145 objects. Within the inner strip of $\pm 5^{\prime}$ the completeness of the catalogue reaches $80 \%$ at the flux limit $S_{3.9}=7.5 \mathrm{mJy}$ and is almost $100 \%$ at $15 \mathrm{mJy}$ (Parijskij et al. 1991). Such flux limits are really quite faint and allow one to identify a large number of steep spectrum sources, if a low frequency catalogue with sufficiently faint flux limit is available. The UTRAO (Douglas et al. 1996) is such a catalogue with a flux limit of $\sim 100 \mathrm{mJy}$ at $365 \mathrm{MHz}$ (see Fig. 1). The RATAN-600 catalogue (RC) provided the first sample which allowed one to calculate the spectral index for practically all UTRAO sources within the region covered by the "Kholod" experiment (Soboleva et al. 1994). Of the original sample of 840 sources (Parijskij et al. 1991), 491 sources matched those of the UTRAO catalogue. Soboleva et al. (1994) could identify optically from POSS (Palomar Optical Sky Survey) 240 sources at galactic latitude $>20^{\circ}$.

\subsection{Construction and properties of the RC/USS sample}

The present study is concerned with sources in the range $4^{\mathrm{h}}<\mathrm{RA}<22^{\mathrm{h}}$ (Parijskij et al. 1991). The first RC/USS sample consists of 40 steep spectrum $\left(\alpha>0.9, f_{\nu} \propto\right.$ $\nu^{-\alpha}$ ), double or triple FRII sources, and optically fainter than the POSS limit. The radio morphology comes from observations with the VLA (Kopylov et al. 1995a). The largest angular size of the radio source (LAS) was not 
Table 1. RC/USS source parameters. The IAU name is in the first column followed by the equatorial, then galactic coordinates and galactic extinction in $R$-band. The radio spectral index is in the seventh column, followed by $3.9 \mathrm{GHz}$ flux density and the LAS of the radio source. The results of optical identification are in the last column. The data have been taken from Kopylov et al. (1995a,b) and Parijskij et al. (1996a)

\begin{tabular}{|c|c|c|c|c|c|c|c|c|c|}
\hline Name & $\begin{array}{l}\text { R.A. } \\
\text { B1950 }\end{array}$ & $\begin{array}{l}\text { Decl } \\
\text { B1950 }\end{array}$ & $\begin{array}{l}l \\
\circ\end{array}$ & $\begin{array}{l}b \\
\circ\end{array}$ & $A_{R}$ & $\alpha_{3900}^{365}$ & $\begin{array}{r}S_{3900} \\
\text { mJy }\end{array}$ & $\begin{array}{r}\text { LAS } \\
{\left[{ }^{\prime \prime}\right]}\end{array}$ & $m_{R}$ \\
\hline J0406+0453 & 040348.22 & 43949.7 & 186 & -33 & 0.41 & 1.02 & 79 & 21.8 & 24.9 \\
\hline $\mathrm{J} 0444+0501$ & 044138.68 & 45555.79 & 192 & -25 & 0.41 & 1.09 & 69 & 10.8 & 23.0 \\
\hline $\mathrm{J} 0457+0452$ & 045515.09 & 44913.77 & 194 & -22 & 0.23 & 1.12 & 56 & 34 & 19.4 \\
\hline J0459+0456 & 045625.51 & 45130.45 & 194 & -22 & 0.23 & 0.95 & 76 & 63.8 & 22.1 \\
\hline J0506+0508 & 050345.56 & $\begin{array}{lll}504 & 21.1\end{array}$ & 195 & -20 & 0.27 & 0.88 & 70 & 0.8 & 21.6 \\
\hline $\mathrm{J} 0552+0451$ & 055016.92 & 44649.9 & 201 & -10 & 1.51 & 1.18 & 65 & 1.6 & $>25.5$ \\
\hline $\mathrm{J} 0743+0455$ & 074036.54 & $\begin{array}{lll}503 & 02.88\end{array}$ & 214 & 13 & 0.12 & 1.07 & 37 & 20.5 & 23.5 \\
\hline J0756+0450 & 075331.2 & 44717.1 & 216 & 16 & 0.07 & 1.16 & 14 & $<1$ & $>25.0$ \\
\hline J0837+0446 & 083451.28 & 45451.82 & 221 & 25 & 0.07 & 1.0 & 54 & 3.9 & 22.4 \\
\hline $\mathrm{J} 0845+0444$ & 084253.28 & 45352.9 & 222 & 27 & 0.09 & 1.14 & 135 & 4.6 & 21.4 \\
\hline J0909+0445 & 090713.51 & 45637.0 & 225 & 32 & 0.08 & 1.0 & 64 & 1 & 20.6 \\
\hline J0934+0505 & 093148.21 & $\begin{array}{lll}517 & 10.76\end{array}$ & 229 & 38 & 0.06 & 1.07 & 36 & 5 & 24.4 \\
\hline $\mathrm{J} 1031+0443$ & 102843.01 & 45833.53 & 240 & 49 & 0.06 & 1.2 & 191 & 33 & 22.5 \\
\hline $\mathrm{J} 1043+0443$ & $1041 \quad 10.27$ & 45612.62 & 243 & 52 & 0.06 & 1.14 & 37 & 48 & 23.0 \\
\hline $\mathrm{J} 1113+0436$ & 111124.05 & 45420.12 & 252 & 57 & 0.10 & 0.98 & 52 & 29 & 22.4 \\
\hline $\mathrm{J} 1152+0449$ & 114949.71 & 50456.75 & 268 & 63 & 0.07 & 1.0 & 29 & 7 & $>24.0$ \\
\hline $\mathrm{J} 1155+0444$ & 115245.43 & $\begin{array}{lll}50013.86\end{array}$ & 269 & 63 & 0.07 & 1.0 & 54 & 13 & 18.6 \\
\hline $\mathrm{J} 1219+0446$ & 121706.94 & $\begin{array}{l}50402.84 \\
\end{array}$ & 282 & 66 & 0.06 & 1.23 & 23 & 118 & 22.0 \\
\hline $\mathrm{J} 1235+0435$ & 123316.52 & 44926.7 & 292 & 67 & 0.06 & 0.98 & 45 & 7 & 21.5 \\
\hline $\mathrm{J} 1322+0449$ & 131931.84 & 50428.13 & 322 & 66 & 0.06 & 0.96 & 47 & 7 & 20.4 \\
\hline $\mathrm{J} 1333+0451$ & 133032.35 & $\begin{array}{l}50708.5 \\
\end{array}$ & 328 & 65 & 0.05 & 1.3 & 11 & 1 & 23.4 \\
\hline $\mathrm{J} 1333+0452$ & 133054.66 & $\begin{array}{lll}507 & 21.17\end{array}$ & 328 & 65 & 0.05 & 1.4 & 16 & 54 & 23.3 \\
\hline $\mathrm{J} 1339+0445$ & 133706.5 & 51015.85 & 332 & 64 & 0.06 & 1.07 & 41 & 34 & 22.7 \\
\hline $\mathrm{J} 1347+0441$ & 134437.58 & 45716.48 & 336 & 63 & 0.06 & 0.98 & 43 & 1.4 & 23.5 \\
\hline $\mathrm{J} 1429+0501$ & 142645.73 & 51443.41 & 353 & 57 & 0.06 & 0.92 & 82 & 11.1 & $>24.0$ \\
\hline $\mathrm{J} 1436+0501$ & 143404.66 & $\begin{array}{llll}5 & 15 & 10.8\end{array}$ & 356 & 56 & 0.06 & 1.25 & 48 & 15 & 22.9 \\
\hline $\mathrm{J} 1439+0455$ & 143715.64 & 50838.68 & 357 & 55 & 0.06 & 1.15 & 40 & 17.9 & $>24.0$ \\
\hline $\mathrm{J} 1510+0438$ & 150743.00 & 45051.72 & 4 & 50 & 0.06 & 0.9 & 67 & 3.4 & 22.1 \\
\hline $\mathrm{J} 1609+0456$ & 160654.69 & 50750.48 & 16 & 38 & 0.14 & 1.15 & 30 & 6.3 & $>24.5$ \\
\hline $\mathrm{J} 1626+0448$ & 162421.72 & 45533.4 & 19 & 34 & 0.18 & 1.26 & 39 & 2.4 & 22.9 \\
\hline $\mathrm{J} 1646+0501$ & 164424.94 & 50628.92 & 22 & 29 & 0.27 & 0.92 & 54 & 15.7 & 21.2 \\
\hline $\mathrm{J} 1658+0454$ & 165543.34 & 45804.9 & 23 & 27 & 0.27 & 1.25 & 31 & $<0.3$ & $>24.5$ \\
\hline $\mathrm{J} 1703+0502$ & 170101.3 & 50620.0 & 24 & 26 & 0.27 & 1.18 & 175 & 1.8 & 23.6 \\
\hline $\mathrm{J} 1720+0455$ & 171736.0 & 45648.0 & 26 & 22 & 0.27 & 1.22 & 19 & $<0.5$ & 20.6 \\
\hline $\mathrm{J} 1725+0457$ & 172304.58 & 50005.0 & 27 & 21 & 0.27 & 1.26 & 27 & 1 & $>24.0$ \\
\hline $\mathrm{J} 1735+0454$ & 173313.52 & 45707.37 & 28 & 19 & 0.39 & 1.0 & 30 & 4 & 23.5 \\
\hline $\mathrm{J} 1740+0502$ & 173806.03 & 50411.1 & 29 & 18 & 0.41 & 1.2 & 32 & 4 & 22.5 \\
\hline $\mathrm{J} 2013+0508$ & 201054.69 & 50124.78 & 47 & -15 & 0.46 & 0.96 & 51 & 10 & 21.1 \\
\hline $\mathrm{J} 2036+0451$ & 203427.46 & 43922.7 & 50 & -20 & 0.27 & 1.02 & 75 & 56 & 19.0 \\
\hline $\mathrm{J} 2144+0513$ & 214156.65 & 45726.1 & 61 & -34 & 0.18 & 1.06 & 72 & $<5.5$ & 18.8 \\
\hline
\end{tabular}

used as a criterion, because only eight sources had LAS larger than $30^{\prime \prime}$. The median LAS of the sample is $7^{\prime \prime}$. The median $365 \mathrm{MHz}$ flux density is $0.5 \mathrm{Jy}$ (average $0.7 \mathrm{Jy}$ ) ranging from $0.2 \mathrm{Jy}$ to $3 \mathrm{Jy}$.

Optical identifications were made from deep observations at the $6 \mathrm{~m}$ telescope, down to about $m_{R}=24$. These results and the optical fields around the sources have been reported by Kopylov et al. (1995b, here after K95b). Table 1 contains information on the basic RC/USS sample: source name, equatorial and galactic coordinates, spectral index, flux, LAS and $m_{R}$. From this list we selected objects which are not unreasonably faint $\left(m_{R}<\right.$ $24 \mathrm{mag}$ ) for a medium sized telescope.
Figure 2 gives a representative $m_{R}-z$ Hubble diagram for radio galaxies collected from the literature, together with the magnitude distribution of the RC/USS objects. The Hubble diagram allows one to estimate a lower limit to redshift, because of the rather sharp lower envelope, especially above $m_{R}=21$. Where the bulk of the $\mathrm{RC} /$ USS galaxies are situated, redshift is expected to be $\gtrsim 0.7$ as shown in Fig. 2. Soboleva et al. (1994) estimated the maximum photometric redshifts for the RC/USS objects from the requirement that radio luminosity is not higher than optical luminosity: when radio flux is known, the minimum optical magnitude may be calculated, hence the rough maximum $z_{\mathrm{ph}}$, which is usually large, $>1$. 


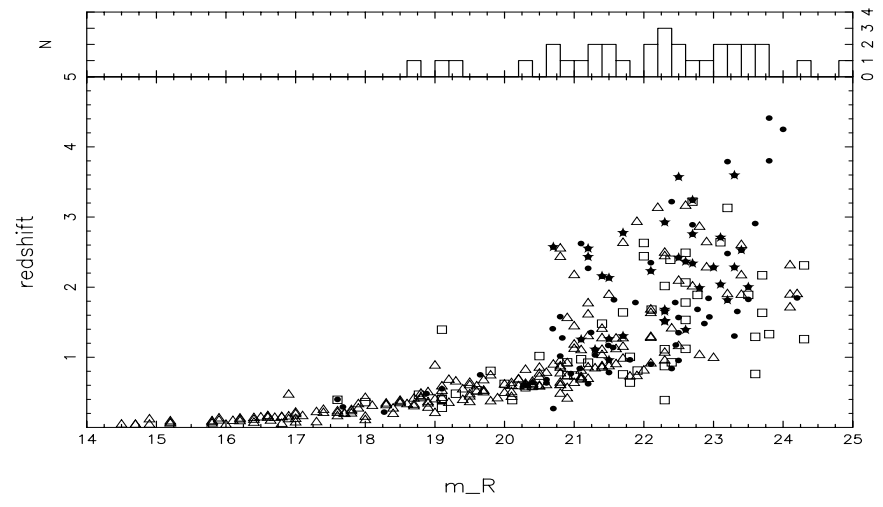

Fig. 2. Hubble diagram in $R$-band for various radio galaxies from the literature. The triangles are from the complete Molonglo sample (McCarthy et al. 1996), the open boxes are from Allington-Smith et al. (1988), Maxfield et al. (1995), McCarthy et al. (1987), McCarthy et al. (1990), McCarthy et al. (1991), Thompson et al. (1994), Windhorst et al. (1991). Filled dots are from Carilli et al. (1997), Chambers et al. (1988), Djorgovski et al. (1988), Dunlop \& Peacock (1993), Eales et al. (1993), Hammer \& LeFevre (1990), Kristian et al. (1978), Lacy et al. (1994), LeFevre et al. (1988), LeFevre \& Hammer (1988), Lilly (1988), Lilly (1989), Owen \& Keel (1995), Miley et al. (1992), Spinrad et al. (1995) and filled stars are from the ESO/Key-Project (Röttgering et al. 1995; Röttgering et al. 1996; van Ojik et al. 1994). Open symbols are $r$-magnitudes, which are transformed as $R=r-0.4$. The histogram of RC/USS sources $R$-magnitudes is shown above. The magnitudes are from K95b. Present NOT-observations are concerned with $R \lesssim 24$

It should be mentioned that one optically bright $\left(m_{R}=19\right)$ object RC $2036+0451$ was measured at the $6 \mathrm{~m}$ telescope to have $z=2.95$ (Pariskij et al. 1996b). Though for a quasar, this large $z$ also supports the view that present selection criteria lead to high average redshift.

The aim of the NOT imaging was to study the morphology of the RC/USS sources with high resolution and confirm the optical identifications. This paper is organised as follows. In Sect. 2 we describe our observations and data reduction. Morphology of individual galaxies is discussed in Sect. 3. The results are summarised in Sect. 4 .

\section{Observations and reductions}

\subsection{Observations}

Optical images were obtained with the $2.56 \mathrm{~m}$ Nordic Optical Telescope (NOT) at La Palma during three observing runs in March, May and December 1994. Table 2 summarises the instrumentation used. In addition, we have some supplementary observations from other observing runs. $I$-band observations of RC $1510+0438$ were made with "Stockholm" CCD in July 1994 and RC 2013+0508 was observed with Brocam1 in September 1994. The complete log of observations is given in Table 3. For each ob- served object it contains the filter used, number of separate images, total integration time, seeing, and date. Calibration stars from Landolt (1992) were observed several times each night at a range of air masses.

In this paper, we shall restrict the discussion to observations made under excellent or good seeing ( $F W H M \lesssim$ 1 ".1) conditions, totaling 22 objects. We present only $R$-band images except for a few cases where the morphology has a strong wavelength dependence and the $\mathrm{S} / \mathrm{N}$ ratio in other passbands is high enough. All observations presented were made under photometric conditions.

"Blooming" of the CCD was a serious problem with the IAC CCD. Some objects were close to bright stars which limited the longest possible exposure time, or a bright star had to be moved outside the CCD. RC 1735+0454 lies close to the galactic plane, hence the field is crowded with bright stars. We could not obtain long exposures of this faint object and had to move it close to the edge of the CCD. The bright star northeast from the centre of gravity of RC $1219+0446$ hampered the observations and the northern part of the radio source was not observed.

\subsection{Reductions}

The reductions were carried out using standard IRAF routines (bias subtraction, trimming, flat fielding). The average bias frame was constructed for each night. The flatfielding was made by twilight flats obtained each evening and morning. All the scientific frames were flattened at better than a 1\%-level. The exposures of each object were registered in position using several stars in the field and then averaged. The number of reference stars varied from three up to a dozen.

The astrometric calibration was carried out using the APM Catalogue (Irwin et al. 1994) whenever possible. For the objects near the galactic plane the Guide Star Catalog (GSC) (Lasker et al. 1990) was used. Due to the small field of view of the CCDs there were typically only a few reference stars in the frame. The number of stars and hence the accuracy of the astrometry strongly depends on galactic latitude. We estimate the accuracy of the astrometric calibration to be typically better than $1 \mathrm{~s}$ of arc. This is enough for the current study, because the typical resolution of the radio map is about $1^{\prime \prime} .5$ and most of the radio sources are so compact that the optical identification is straightforward.

As a check of our photometry in the March and May 1994 run we measured comparison stars of OJ 287 (Fiorucci \& Tosti 1996). The derived brightnesses were consistent with each other within 0.1 magnitudes.

\section{Reduced images: Overview of the morphology}

In this section we give a greyscale image and a contour map for each identified object (Fig. 3) The close environment and faint features can be studied from the greyscale 
Table 2. Instruments

\begin{tabular}{lllll}
\hline CCD & Date & Field size (pixels) & Field size $\left(^{\prime}\right)$ & Pixel size $\left(^{\prime \prime}\right)$ \\
\hline Astromed & & & & \\
EEV P88200 & March 1994 & $1152 \times 770$ & $3.1 \times 2.1$ & 0.163 \\
IAC CCD & May 1994 & $1024 \times 1024$ & $2.4 \times 2.4$ & 0.14 \\
$\begin{array}{l}\text { THX31156 } \\
\text { Brocam 1 }\end{array}$ & & & \\
TK1024A & December 1994 & $1024 \times 1024$ & $3 \times 3$ & 0.176 \\
\hline
\end{tabular}

Table 3. Journal of observations

\begin{tabular}{|c|c|c|c|c|c|c|c|c|c|c|c|}
\hline Object & Band & $\begin{array}{l}\text { No. of } \\
\text { images }\end{array}$ & $\begin{array}{r}T_{\mathrm{int}} \\
{[\mathrm{sec}]}\end{array}$ & $\begin{array}{l}\text { Seeing } \\
{\left[{ }^{\prime \prime}\right]}\end{array}$ & $\begin{array}{l}\text { Date } \\
1994\end{array}$ & Object & Band & $\begin{array}{l}\text { No. of } \\
\text { images }\end{array}$ & $\begin{array}{r}T_{\mathrm{int}} \\
{[\mathrm{sec}]}\end{array}$ & $\begin{array}{l}\text { Seeing } \\
{\left[{ }^{\prime \prime}\right]}\end{array}$ & $\begin{array}{l}\text { Date } \\
1994\end{array}$ \\
\hline \multirow[t]{2}{*}{$\mathrm{RC} 0444+0501$} & $R$ & 5 & 3300 & 2.0 & 3.12 . & RC $1219+0446$ & $R$ & 5 & 3000 & 0.5 & 18.5 \\
\hline & $I$ & 3 & 2700 & 2.0 & 4.12 . & $\mathrm{RC} 1235+0435$ & V & 5 & 3000 & 3 & 14.3 \\
\hline \multirow[t]{4}{*}{$\mathrm{RC} 0457+0452$} & $B$ & 3 & 3200 & $3.2-3.9$ & 3. \& 5.12 . & & $V$ & 3 & 2100 & 0.6 & 17.5 \\
\hline & V & 2 & 1800 & 0.7 & 2.12 & & $R$ & 3 & 1800 & 3 & 14.3 \\
\hline & $R$ & 4 & 3600 & 0.8 & 2.12 . & & $R$ & 3 & 1800 & 0.6 & 17.5 \\
\hline & $I$ & 4 & 2100 & $2.0-2.9$ & 4. \& 5.12 . & RC $1322+0449$ & V & 6 & 3600 & 1.8 & 14.3 \\
\hline \multirow[t]{3}{*}{$\mathrm{RC} 0459+0456$} & $V$ & 2 & 1800 & 0.7 & 2.12 . & & $R$ & 3 & 1800 & 1.8 & 14.3 \\
\hline & $R$ & 2 & 1800 & 0.6 & 2.12 . & RC $1333+0451$ & $V$ & 1 & 600 & 0.6 & 18.5 \\
\hline & $I$ & 3 & 2700 & 2.0 & 3.12 . & & $R$ & 3 & 2100 & 0.5 & 18.5 \\
\hline \multirow[t]{3}{*}{$\mathrm{RC} 0506+0558$} & V & 2 & 1200 & 0.8 & 2.12 . & RC $1339+0445$ & $V$ & 5 & 3000 & 1.4 & 15.3 \\
\hline & $R$ & 6 & 1800 & 0.8 & 2.12 . & & $R$ & 3 & 1800 & 1.4 & 15.3 \\
\hline & $I$ & 2 & 1200 & 1.5 & 4.12 . & RC $1347+0441$ & $V$ & 5 & 3300 & 1.1 & 16. \\
\hline \multirow[t]{3}{*}{$\mathrm{RC} 0743+0455$} & $V$ & 3 & 1800 & 1.3 & 15.3 & & $R$ & 3 & 2400 & 0.7 & 16.5 \\
\hline & $R$ & 3 & 1800 & 1.1 & 15.3 & RC $1510+0438$ & $V$ & 3 & 2100 & 0.6 & 17.5 \\
\hline & $R$ & 1 & 600 & 0.8 & 2.12 . & & $R$ & 7 & 4100 & 0.5 & $17 \& 18.5$ \\
\hline \multirow[t]{3}{*}{$\mathrm{RC} 0837+0446$} & $V$ & 3 & 1800 & 0.9 & 14.3 & RC $1609+0456$ & $V$ & 1 & 600 & 0.6 & 18.5 \\
\hline & $R$ & 4 & 2400 & 1.0 & 14.3 & & $R$ & 2 & 600 & 0.5 & 18.5 \\
\hline & $I$ & 3 & 2400 & 1.6 & 4.12 . & RC $1626+0448$ & $V$ & 2 & 1200 & 1.7 & 15.3 \\
\hline \multirow[t]{3}{*}{$\mathrm{RC} 0845+0444$} & $V$ & 1 & 600 & 1.2 & 15.3 & & $R$ & 2 & 1200 & 1.7 & 15.3 \\
\hline & $R$ & 1 & 600 & 1.1 & 15.3 & RC $1646+0501$ & $V$ & 3 & 1800 & $1.5-2.0$ & 13.3 \\
\hline & $I$ & 2 & 2800 & 1.2 & 4.12 . & & $R$ & 3 & 1800 & $1.5-2.0$ & 13.3 \\
\hline \multirow[t]{8}{*}{ RC $909+0445$} & $B$ & 3 & 2700 & 1.8 & 3.12 . & RC $1703+0502$ & $R$ & 4 & 2400 & 0.6 & 17.5 \\
\hline & $B$ & 4 & 4500 & 3.0 & 5.12 . & RC $1720+0455$ & $V$ & 2 & 1200 & $1.5-2.0$ & 13.3 \\
\hline & V & 2 & 1200 & 1.7 & 14.3 & & $V$ & 1 & 600 & 0.7 & 16.5 \\
\hline & $V$ & 2 & 1800 & 1.6 & 3.12 . & & $R$ & 1 & 600 & $1.5-2.0$ & 13.3 \\
\hline & $R$ & 2 & 1200 & 1.7 & 14.3 & & $R$ & 2 & 1200 & 0.7 & 16.5 \\
\hline & $R$ & 4 & 3600 & 2.0 & 3.12 . & $\mathrm{RC} 1735+0454$ & $R$ & 12 & 3080 & 0.6 & $17 \& 18.5$ \\
\hline & $I$ & 1 & 900 & 1.5 & 4.12 . & RC $1740+0502$ & $V$ & 3 & 1800 & 0.6 & 16.5 \\
\hline & $I$ & 3 & 2700 & 3.0 & 5.12 . & & $R$ & 2 & 1200 & 0.6 & 16.5 \\
\hline \multirow[t]{5}{*}{ RC $1031+0443$} & $V$ & 6 & 3600 & $1.5-2.0$ & 13.3 & RC $2013+0508$ & $V$ & 2 & 1800 & 1.5 & 3. \& 4.12 . \\
\hline & $V$ & 3 & 1800 & 1.5 & 15.3 & & $R$ & 1 & 900 & 1.2 & 4.12 . \\
\hline & $R$ & 3 & 1800 & 1.6 & 13.3 & & $R$ & 1 & 600 & 0.6 & 4.9 . \\
\hline & $R$ & 3 & 1800 & 0.9 & 15.3 & RC $2036+0451$ & $B$ & 2 & 1800 & 1.4 & 2.12 . \\
\hline & $I$ & 2 & 1800 & 1.4 & 4.12 . & & $V$ & 2 & 1800 & 1.5 & 3. \& 4.12 . \\
\hline \multirow[t]{2}{*}{ RC $1043+0443$} & $V$ & 5 & 3000 & 1.7 & 14.3 & & $R$ & 1 & 900 & 1.1 & 4.12 . \\
\hline & $R$ & 4 & 2400 & 1.7 & 14.3 & & $I$ & 1 & 900 & 3.0 & 5.12 . \\
\hline \multirow[t]{2}{*}{$\mathrm{RC} 1113+0436$} & $V$ & 5 & 3000 & 1.6 & 15.3 & RC $2144+0513$ & $B$ & 2 & 1800 & 1.8 & 3.12 . \\
\hline & $R$ & 3 & 1800 & 1.6 & 15.3 & & $V$ & 2 & 1800 & 1.0 & 2.12 . \\
\hline \multirow[t]{2}{*}{ RC $1152+0449$} & $V$ & 5 & 3000 & 1.0 & 16.5 & & $R$ & 3 & 1920 & 1.0 & 2.12 . \\
\hline & $R$ & 3 & 1800 & 0.8 & 16.5 & & $I$ & 2 & 1800 & 1.2 & 4.12 . \\
\hline \multirow[t]{2}{*}{ RC $1155+0444$} & $V$ & 1 & 600 & 0.6 & 17.5 & & & & & & \\
\hline & $R$ & 1 & 600 & 0.6 & 17.5 & & & & & & \\
\hline
\end{tabular}


Table 4. NOT imaging data. The diameter of the aperture is indicated in arcseconds. The magnitudes are without correction of galactic extinction. Ellipticity and position angle of resolved sources is measured with the same aperture as the magnitudes. The radio position angles are measured from Kopylov et al. (1995a)

\begin{tabular}{llllllrrr}
\hline Name & Aperture & $m_{R}$ & $m_{\text {err }}$ & $m_{V-R}$ & $m_{\text {err }}$ & $e$ & PA $_{\text {opt }}$ & PA $_{\text {radio }}$ \\
\hline RC 0457+0452 & 7 & 19.72 & 0.01 & 0.93 & 0.05 & 0.13 & 13 & 58 \\
RC 0506+0558 & 3.5 & 21.54 & 0.03 & 1.29 & 0.13 & unresolved &.. & -75 \\
RC 0837+0446 & 6.5 & 22.19 & 0.08 & 0.74 & 0.13 & 0.23 & 76 & -67 \\
RC 0845+0444 & 8.2 & 20.75 & 0.08 & 1.15 & 0.17 & 0.25 & -78 & 9 \\
RC 1031+0443 & 6.5 & 22.32 & 0.12 & 1.12 & 0.23 & 0.57 & 88 & -36 \\
RC 1152+0449 & 3 & 22.23 & 0.07 & 0.84 & 0.15 & 0.11 & -33 & -15 \\
RC 1155+0444 & 8.4 & 18.81 & 0.02 & 1.09 & 0.05 & 0.28 & -54 & -76 \\
RC 1235+0453 & 4.2 & 21.70 & 0.06 & 0.96 & 0.17 & 0.26 & 43 & -50 \\
RC 1347+0441 & 2 & 23.99 & 0.19 & 0.75 & 0.43 & 0.32 & -29 & -49 \\
RC 1510+0438 & 3 & 22.20 & 0.04 & 1.57 & 0.25 & 0.08 & -79 & 62 \\
RC 1703+0502 & 3 & 23.69 & 0.18 &. &. & 0.49 & -86 & -82 \\
RC 1720+0455 & 4.2 & 20.34 & 0.02 & 1.15 & 0.07 & unresolved &.. & point \\
RC 1740+0502 & 3 & 22.19 & 0.08 & 0.74 & 0.12 & 0.05 & 66 & 64 \\
RC 2013+0508 & 5.3 & 20.70 & 0.04 & 0.22 & 0.06 & unresolved &.. & -55 \\
RC 2036+0451 & 4.2 & 19.06 & 0.02 & 0.35 & 0.04 & unresolved &.. & -2 \\
RC 2144+0513 & 3.5 & 18.89 & 0.02 & 0.24 & 0.03 & unresolved &.. & point \\
\hline
\end{tabular}

image and the confidence level of the features and light distribution from the contour map. The field of view of the greyscale image is indicated in the upper left hand corner. The images are slightly smoothed with a Gaussian function ( $F W H M=\frac{1}{2}$ seeing) in order to enhance the low surface brightness features and maintain the resolution of the original images. The centre of gravity of the radio source and the positions of the radio lobes are indicated with a cross and circles, respectively. The images are presented in linear scale from $0 \sigma$ to $10 \sigma$ above the background of the image. In contour maps the object is in the origin and the numbers on both the vertical and horizontal axes refer to distance in arcsecond. The contour interval is $0.5 \mathrm{mag} \operatorname{arcsec}^{-2}$ and the surface brightness of the lowest contour is indicated in the upper right hand corner. The limiting surface brightness at which objects can be detected is typically between 25 and 26 mag $\operatorname{arcsec}^{-2}$. For RC $1510+0438$ we also give $V$ and $I$ band images. Uncertain identifications are presented in Fig. 4 and "faint objects" in Fig. 5.

The magnitudes are based on aperture photometry using DAOPHOT. The size of the aperture was selected in such a way that 1) as much light as possible was included in the aperture while keeping the errors reasonable but 2) the companions were excluded. The image shapes are determined by the moments of the brightness distribution using IMEXAMINE (Eq. (4) in Valdes et al. 1983) with the size of the aperture the same as in photometry. The estimation is vague for small ellipticity (e.g. RC 1152+0449) or when the inner regions have a different position angle than the outer regions (e.g. RC 1031+0443). The results of photometry and image shape analysis are given in Table 4. The magnitudes between this work and K95b are generally in agreement. The differences are primarily caused by the better seeing conditions at NOT, as compared with the $6 \mathrm{~m}$-telescope and the different size of the aperture.

\subsection{Identified objects}

In this section we give notes on individual objects (Fig. 3). For the strongest sources alternative names are given in brackets.

\section{RC $0457+0452$}

This is one of the brightest objects in this sample. The new VLA map shows that the radio source has FRI structure (an unpublished radio map). This agrees with a high optical to radio luminosity ratio (Parijskij et al. 1996a). There is a near companion $\sim 2.5^{\prime \prime}$ northwest from the nucleus. In addition there are four faint companions in the southeast $\left(5^{\prime \prime}\right)$. The position angle of the galaxy is not uniform, which is possibly due the close companions. The inner isophotes are roughly perpendicular to the radio axis and the outermost fuzz is roughly aligned with the radio axis. The same trend can be seen in both $R$ and $V$-band images. This object is possibly situated in a cluster of galaxies and there are a few companions with similar brightness. The companion $20^{\prime \prime}$ to the east has a double nucleus $\left(m_{R}=19.26, m_{V-R}=0.69\right)$, hence it is apparently a merger, and the companion $22^{\prime \prime}$ to the south has distorted morphology $\left(m_{R}=19.94, m_{V-R}=0.93\right)$.

\section{RC 0506+0508}

This is a faint point-like source, even under excellent seeing conditions. It might be a high redshift $\left(z>1, m_{R}<\right.$ -23) quasar because in the quasar catalogue by VeronCetty \& Veron (1996) there are only a few quasars fainter than 21 magnitudes with $z<1$. The companions $14^{\prime \prime}$ to the northwest have a multicomponent structure with an extended diffuse emission. 


\section{RC $0837+0446$}

The galaxy is marginally resolved and lies in or behind a galaxy cluster. In the lower left hand corner of the grey scale image is a trail of a solar system object.

\section{RC $0845+0444$}

The optical counterpart of this radio source coincides with the western radio component. This object is optically extended and there is a faint extension towards the southwest.

\section{RC $1031+0443$}

$(4 \mathrm{C}+05.43 \&$ PKSB1028+049). The galaxy is near the centre of gravity of the radio source. The object is extended and has a multicomponent structure. The strongest optical emission is aligned with the radio source, but the position angle of the outermost isophotes is almost perpendicular to the radio axis.

\section{RC 1152+0449}

This galaxy has two or possibly three components. The outer isophotes of the galaxy are box-like, but the separate components are roughly aligned with the radio axis. The faint companion $4^{\prime \prime}$ to the west is near to the western radio lobe. This blue companion may be related to the radio source $\left(m_{R}=23.0, m_{V-R} \sim 0.3\right)$.

This is the only source where the astrometry of the present work is not consistent with the result of K95b, but coincides with current identification in Parijskij et al. (1996a).

\section{RC 1155+0444}

This is the brightest galaxy in our sample. The galaxy is elliptical and it is clearly aligned with the radio source. The two neighbouring galaxies are possibly interacting foreground galaxies.

\section{RC 1235+0453}

This faint galaxy is spatially extended in our $R$-band images. The fuzz $\sim 1^{\prime \prime}$ northwest from the nucleus has a clumpy structure. Our deep images show faint low surface brightness companions near the object $\sim 10^{\prime \prime}$ to the east, north and west. In the $V$-band images only the core of the galaxy is detected.

\section{RC 1347+0441}

This galaxy is the faintest of our sample. The object is elongated and the size of the optical object is roughly the same as the radio source.

\section{RC 1510+0438}

This is the most spectacular object in our sample, lying in a group of galaxies and having apparently wavelength dependent properties. In the $R$ - and $I$-band image the object is almost round (Table 4 ) but in the $V$-band the object is weakly elongated with the same position angle as the radio source. The redshift estimation from $B V R I$ colours suggest $z \sim 0.6$ (Pariskij et al. 1996b). If this is the case, the strong emission lines [O II] 3727 and [O III] 5007 would be shifted into $R$ and $I$ band, respectively, possibly causing the wavelength dependence of morphology. However, new colours from the $6 \mathrm{~m}$-telescope do not agree with strong line contribution. Another consequence of such redshift would be that this would be one of the faintest (intrinsically) radio galaxies in the Hubble diagram (Fig. 2). There are three relatively bright galaxies and one faint companion galaxy within $5^{\prime \prime}$ of the object. All the companions are bluer than the object $\left(m_{R-I}=1.52\right)$. The objects towards the east, C1 $\left(m_{R}=22.54, m_{V-R}=0.51, m_{R-I}=0.27\right)$, north, C2 $\left(m_{R}=22.34, m_{V-R}=0.81, m_{R-I}=0.87\right)$ and west,C3 $\left(m_{R}=23.24, m_{R-I}=0.83\right)$ could be foreground galaxies. In addition there is a faint companion 1.5 north from the object.

\section{RC $1703+0502$}

(PKS B1701+051). This is one of the strongest and most compact radio sources of the present sample. The optical and radio axes are clearly aligned and the sizes are almost the same. There are a few faint galaxies in the field, but no close companions. This object is possibly located behind a foreground galaxy cluster although some of the field objects might be faint galactic stars.

\section{RC $1720+0455$}

This object is compact in radio and optical. This suggests that it is a QSO and the same conclusion may be drawn from radio-optical luminosity consideration (Parijskij et al. 1996a). The extension towards the southeast, seen in K95b, was an artifact. There are a few companions close to the object. The southern companion either has a double nucleus or a dust lane. The wide field image shows several faint companions, hence this galaxy is either in a cluster of galaxies or behind one.

\section{RC 1740+0502}

This source was identified by K95b and it is marginally resolved in the $R$-band image. In the $V$-band image the object has an extension to the south west in contrast to almost round morphology in $R$-band (see Table 4 ).

\section{RC 2013+0508}

This unresolved object could possibly be a quasar. Because of its low galactic latitude $(b \sim-15)$, the field is crowded with stars.

\section{RC 2036+0451}

(MRC 2034+046). This is the second of the two triple radio sources in this sample. A point source coincides with the central component fairly well. This is the only object with known redshift ( $z=2.95$ Pariskij et al. 1996a). This indicates that the absolute magnitude of this quasar is $m_{R} \approx-29$.

\section{RC 2144+0513}

This object is unresolved. The companion $3^{\prime \prime}$ to the southwest is most likely a galactic star $\left(m_{R}=20.90, m_{V-R}=\right.$ 1.1). The profile of this object matches perfectly with the average stellar profile from the same field. 
RC $0457+0452$
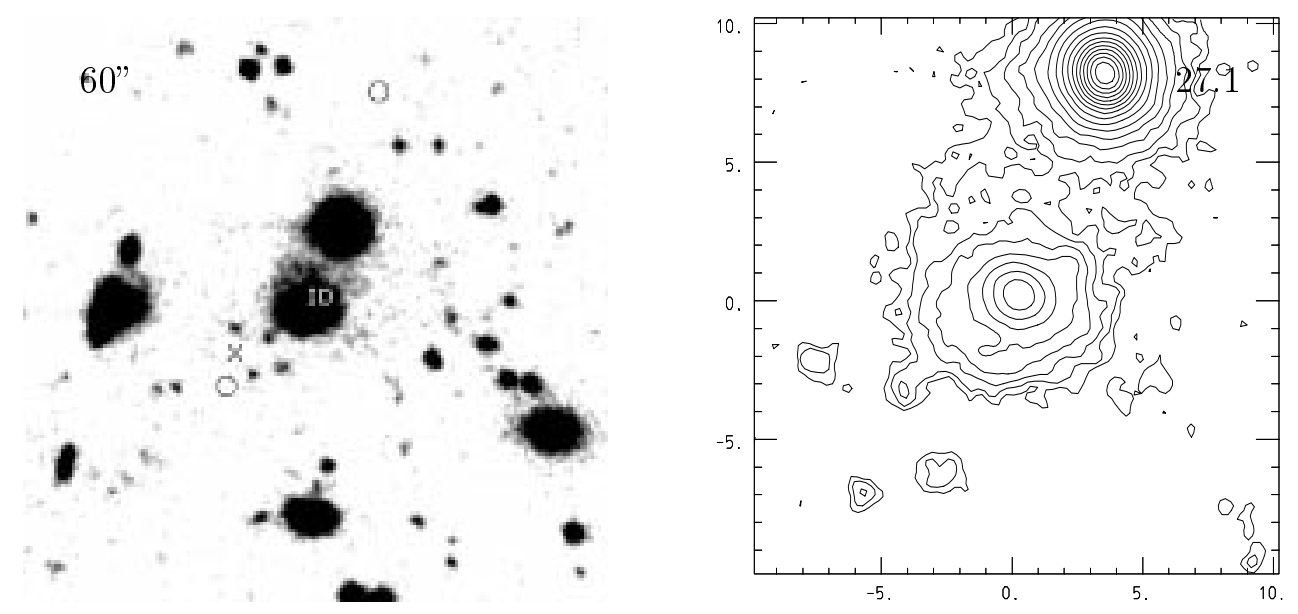

RC $0506+0558$
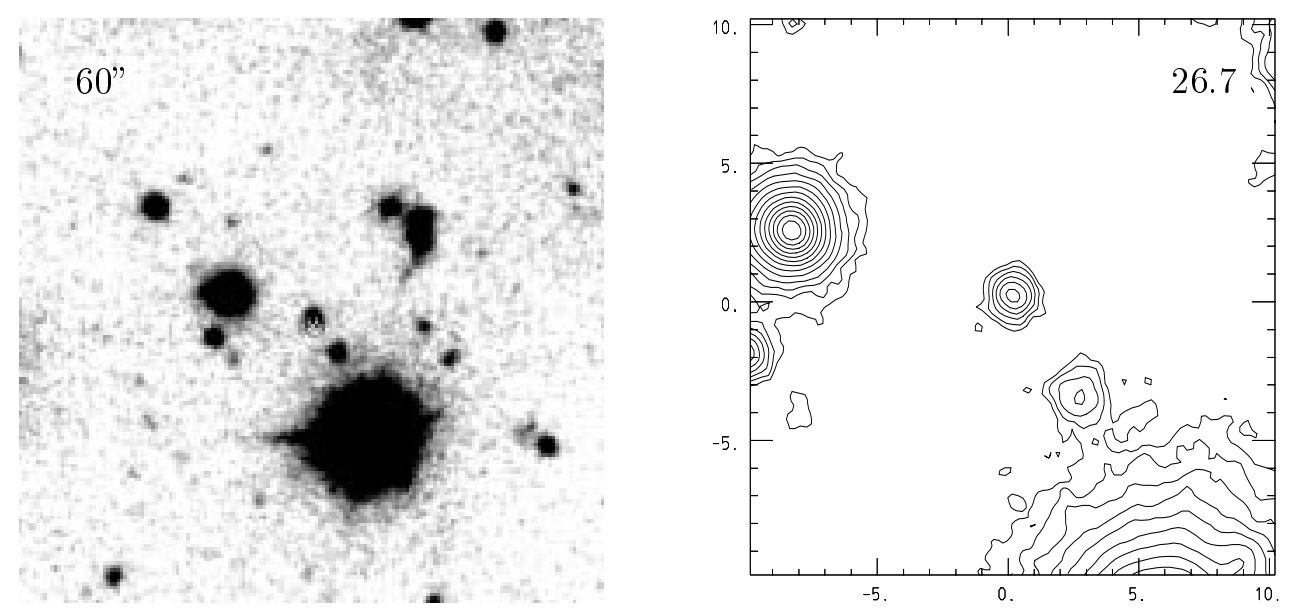

RC $0837+0446$
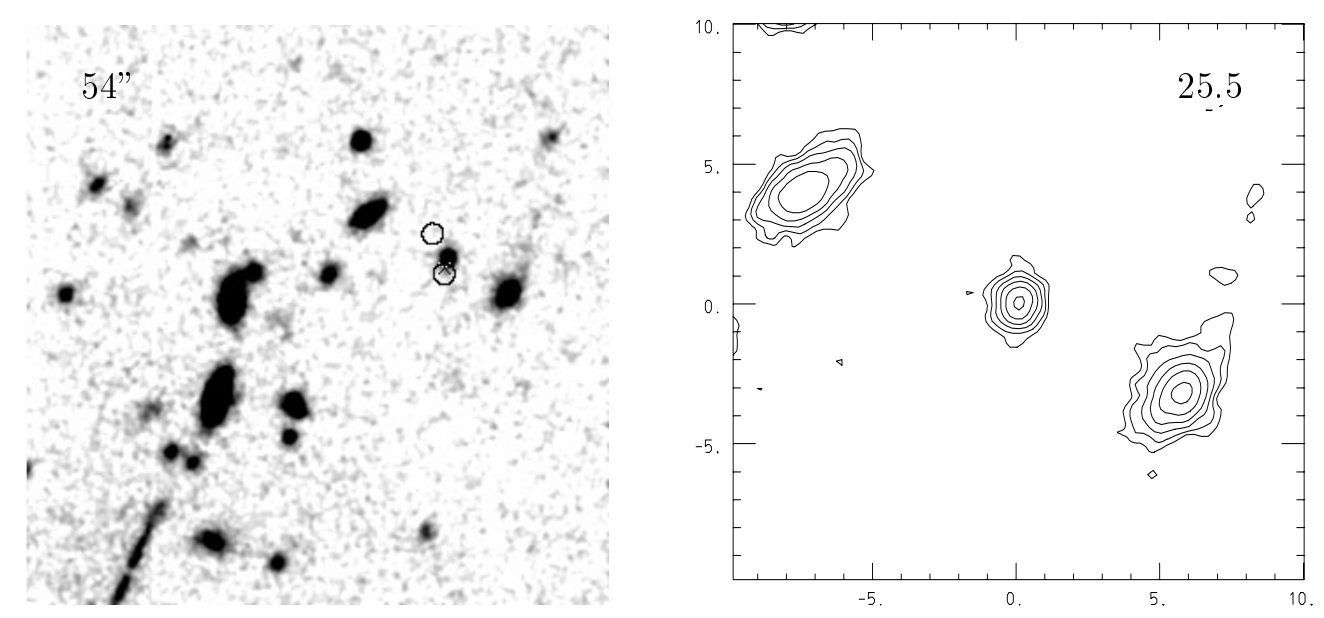

Fig. 3. Optical $R$-band images of identified RC/USS-sources. North is up and east is to the left. The size of the greyscale image is indicated in the upper left hand corner. The position of the radio lobes and centre of gravity are indicated by $1^{\prime \prime}$ diameter circles and a cross, respectively. The contour image is a $20^{\prime \prime} \times 20^{\prime \prime}$ field around the object. The surface brightness of the lowest contour is indicated in the upper right hand corner. The offset in arcseconds relative to the identified object is also indicated. In addition we give $V$ - and $I$-band contour maps for RC $1510+0438$ and $V$-band greyscale image for RC $1740+0502$. See text for comments on each object 
RC 0845+0444
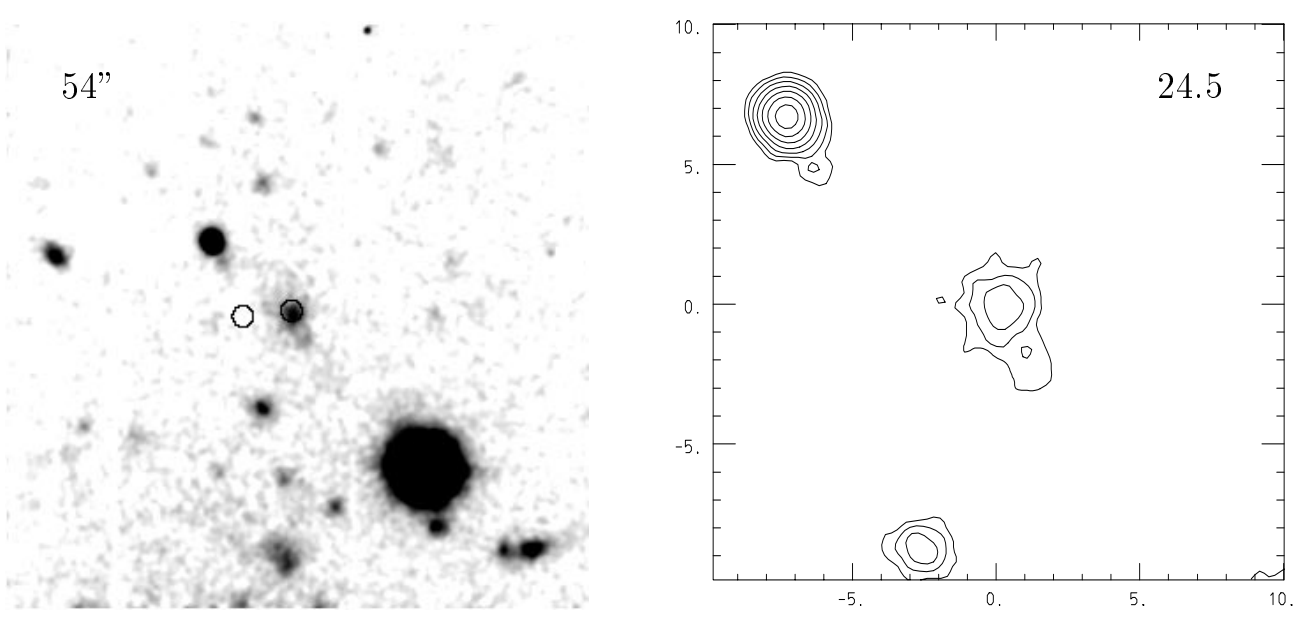

RC 1031+0443
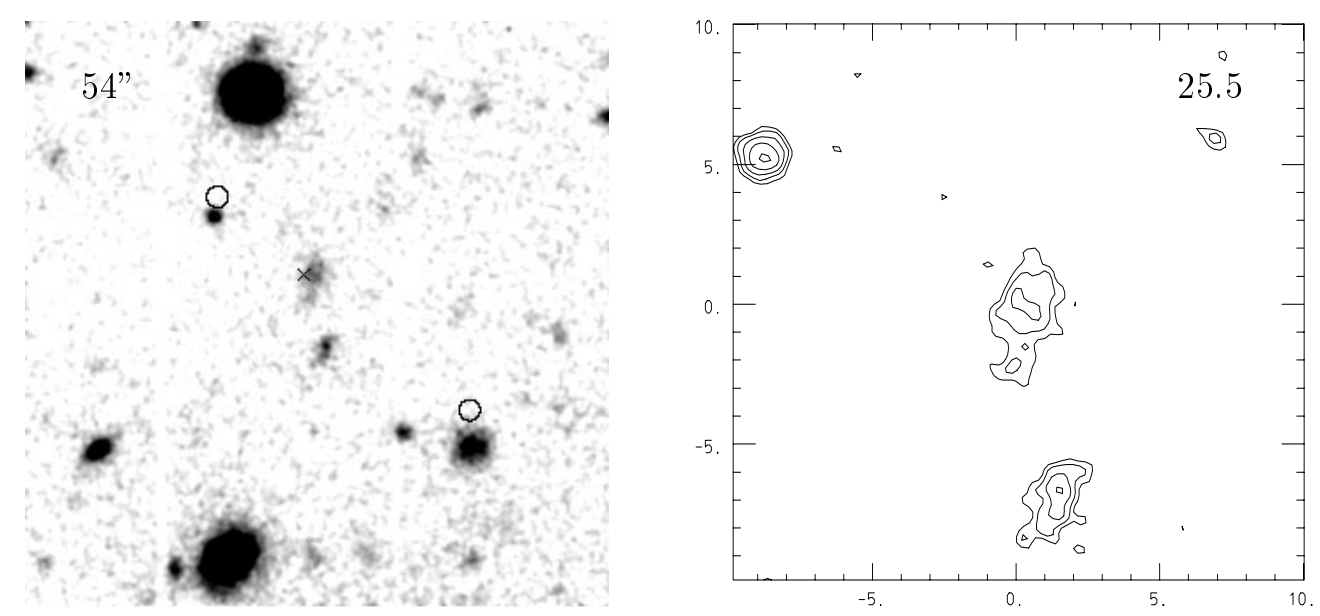

RC $1152+0449$
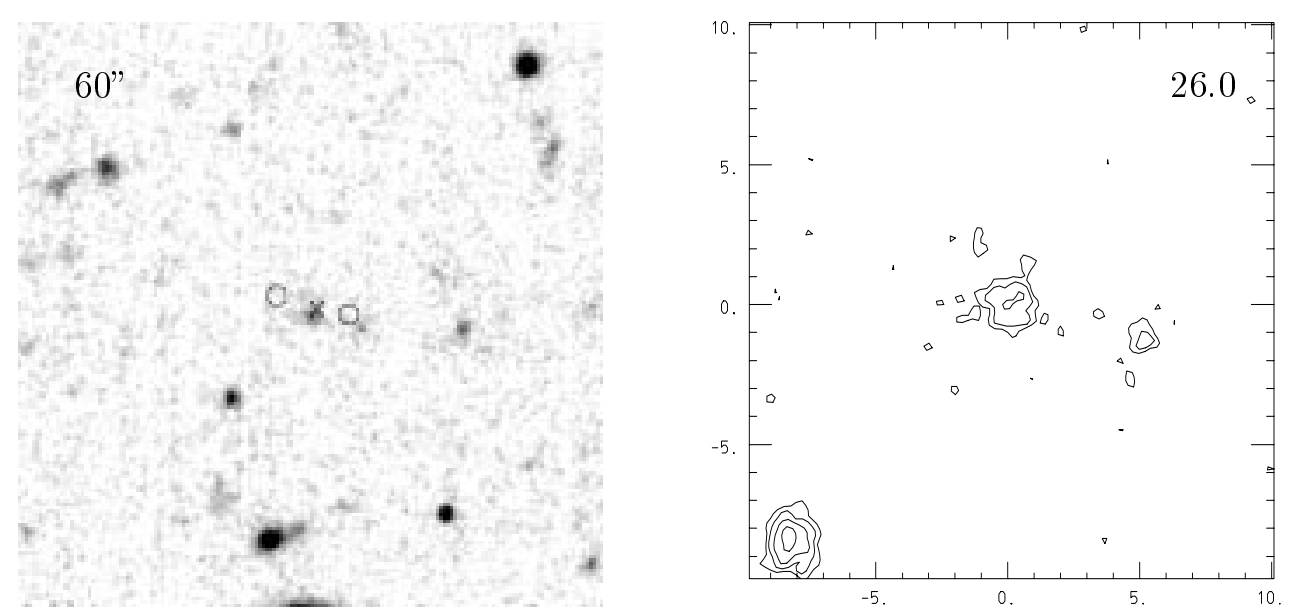

Fig. 3. continued 
RC $1155+0444$
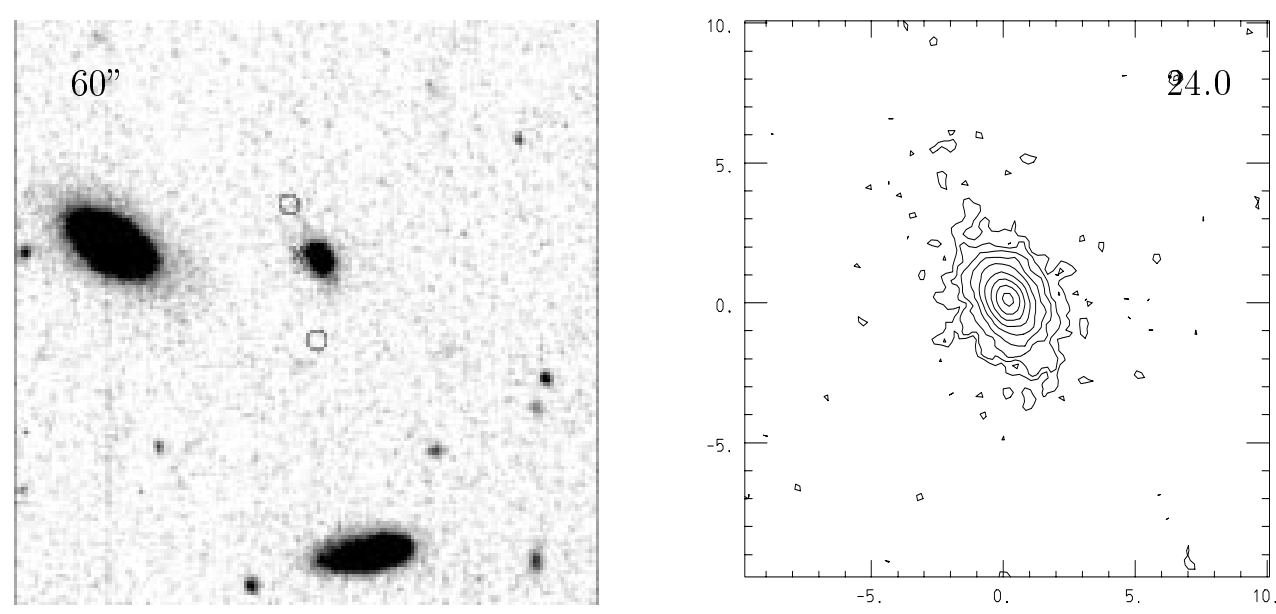

$\mathrm{RC} 1235+0453$
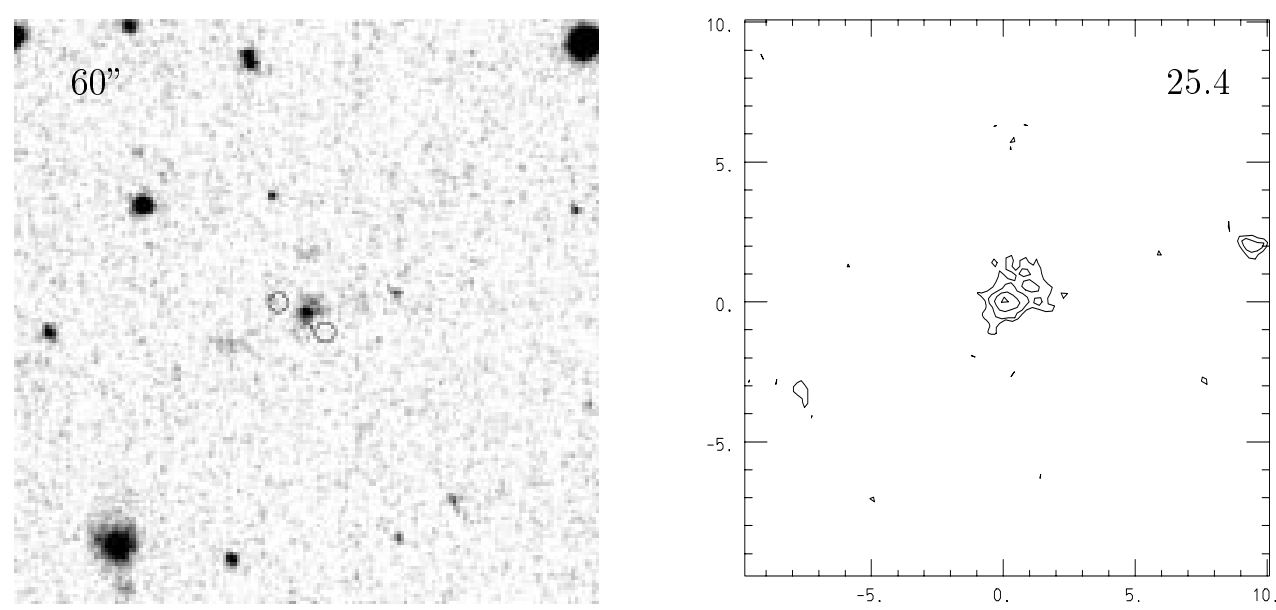

RC $1347+0441$
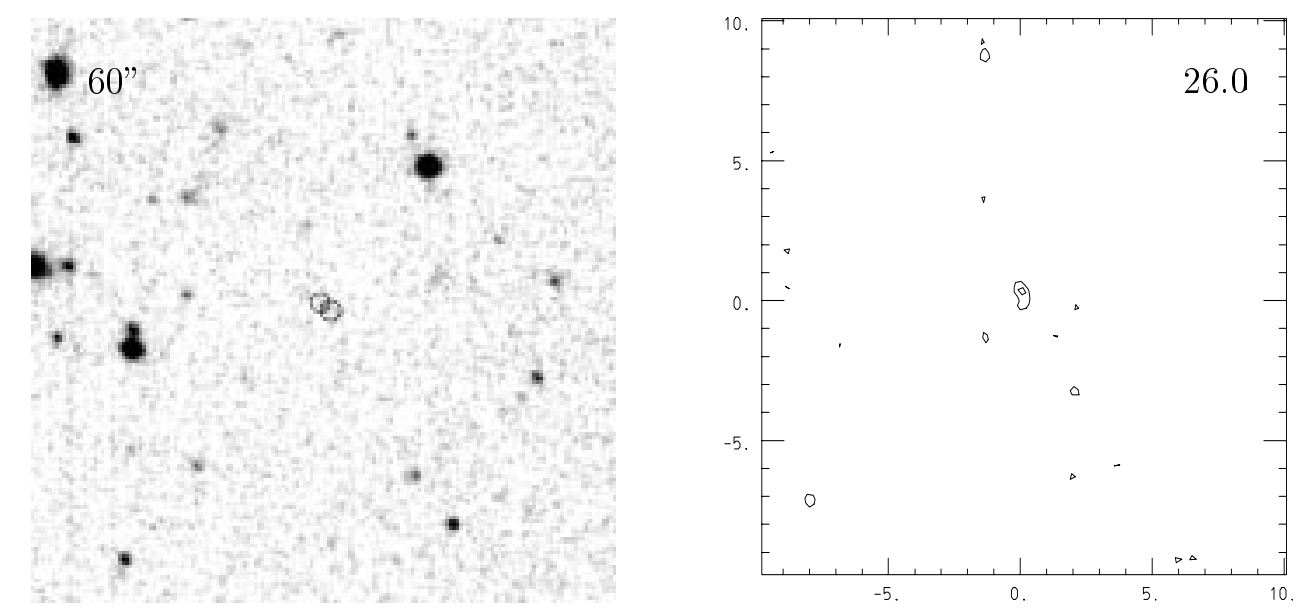

Fig. 3. continued 
RC $1510+0438$
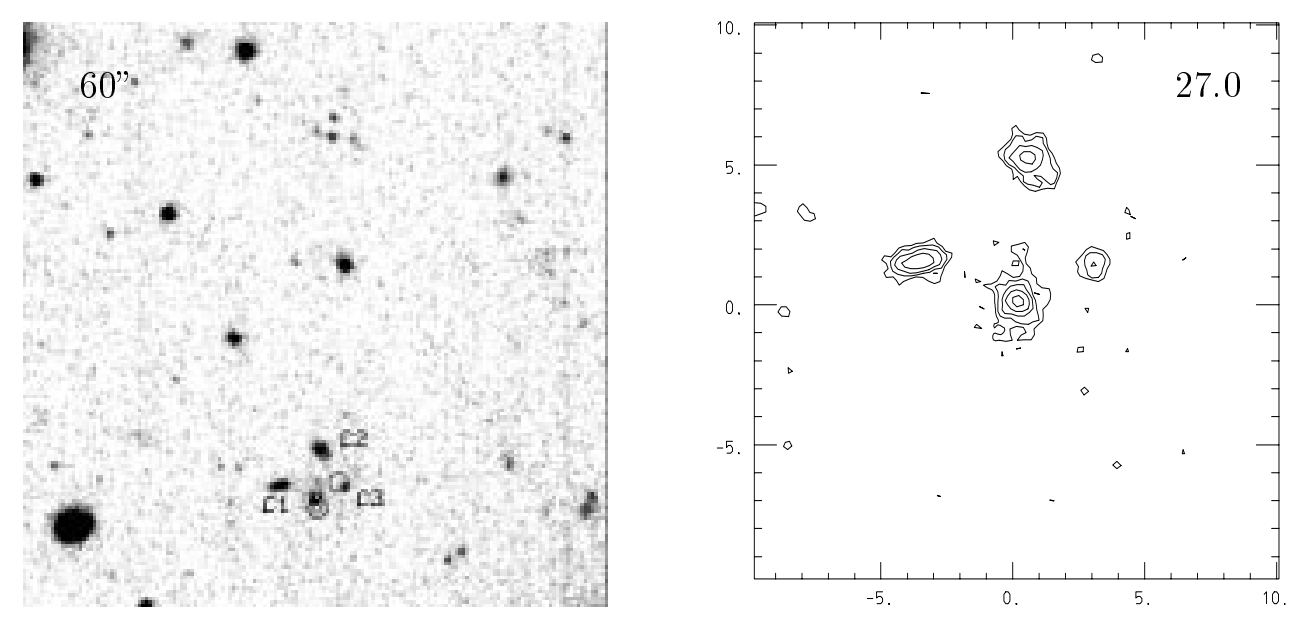

RC $1510+0438$
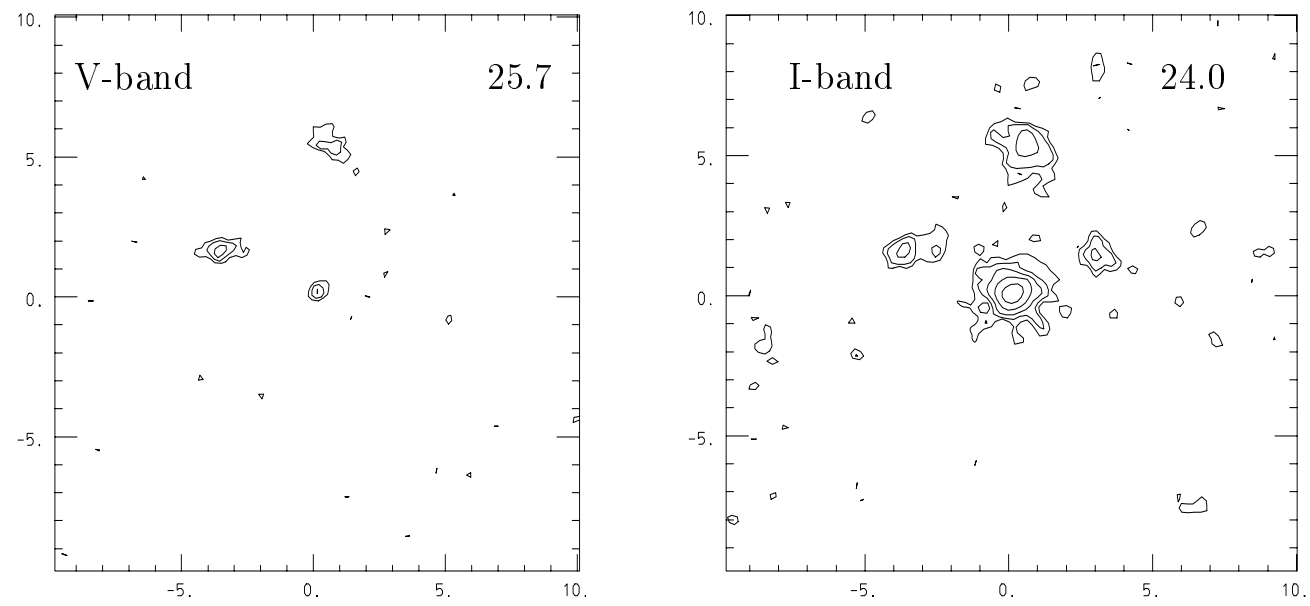

$\mathrm{RC} 1703+0502$
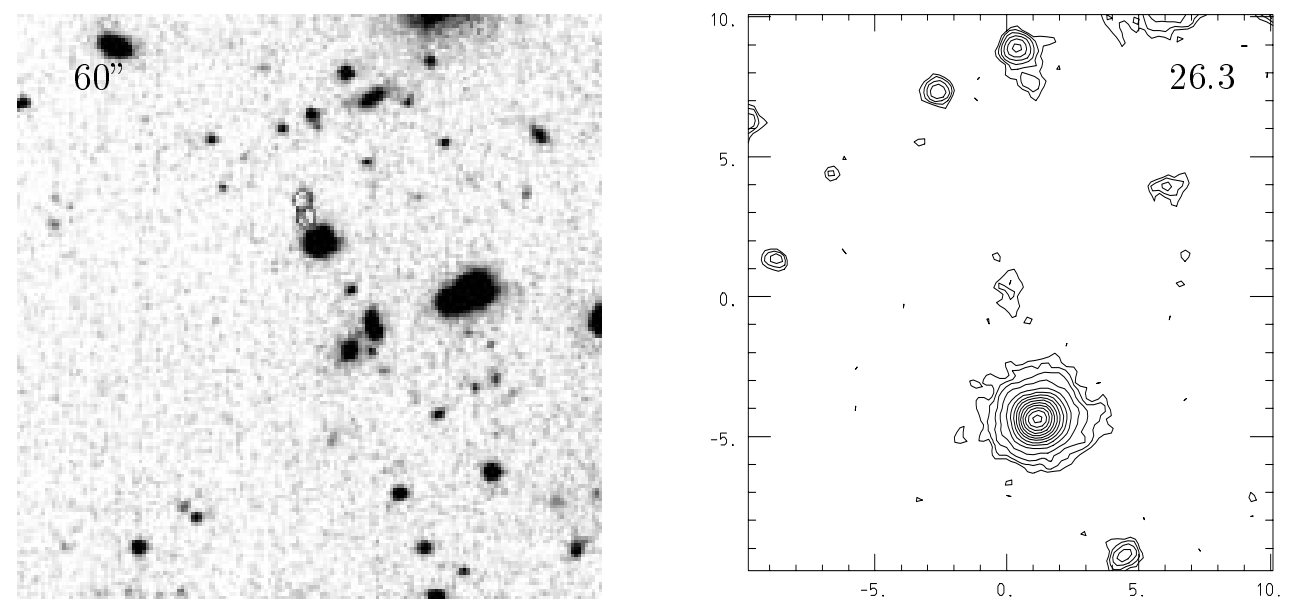

Fig. 3. continued 
RC $1720+0455$
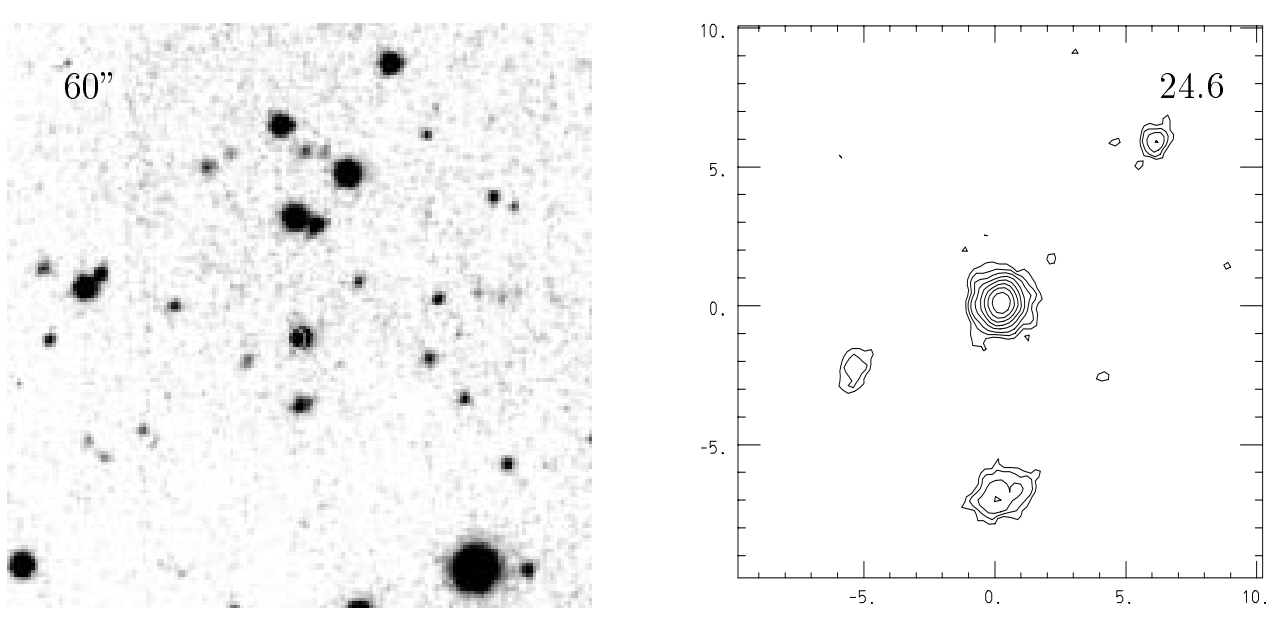

RC $2013+0508$
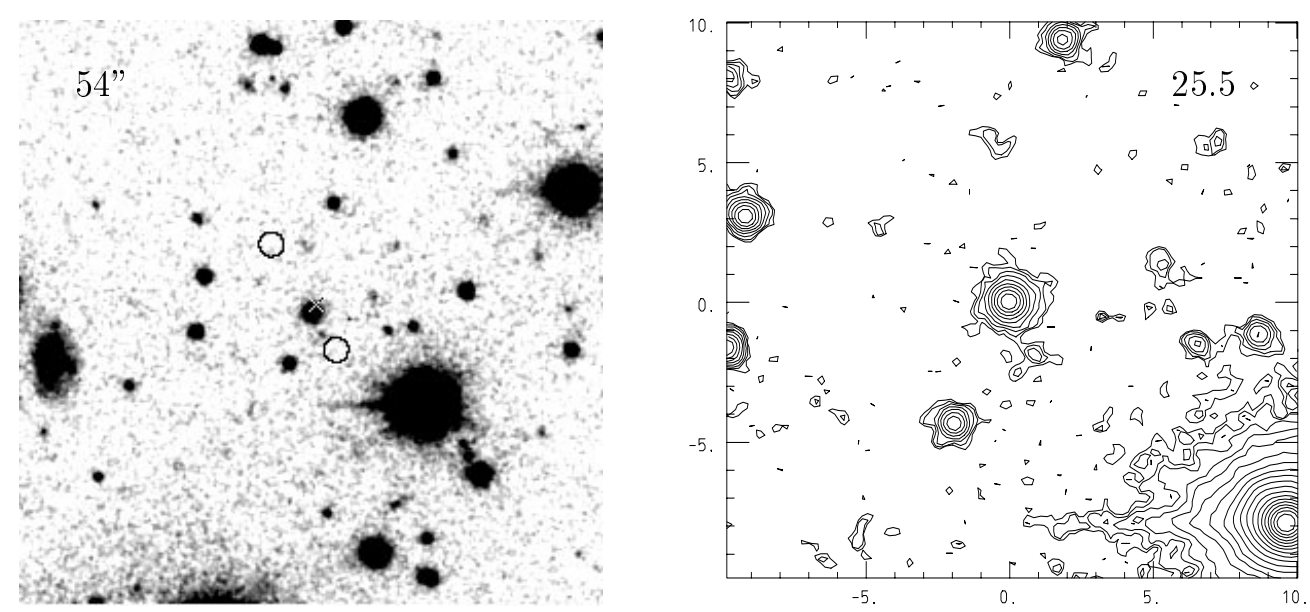

RC 2036+0502
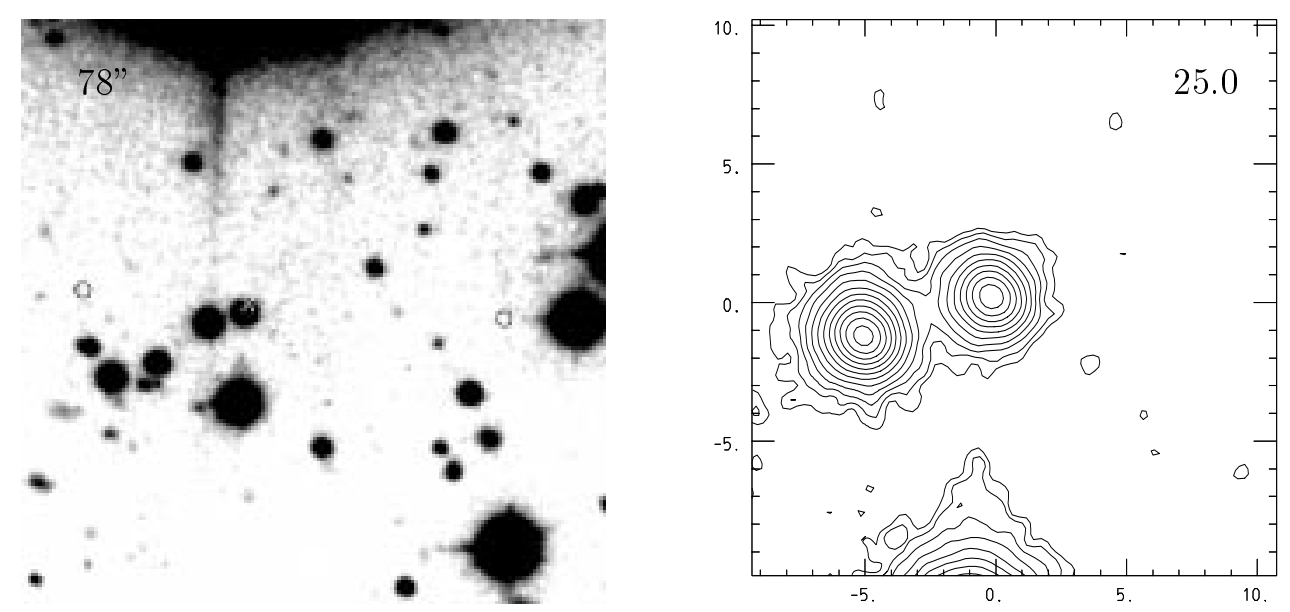

Fig. 3. continued 
RC 2144+0513
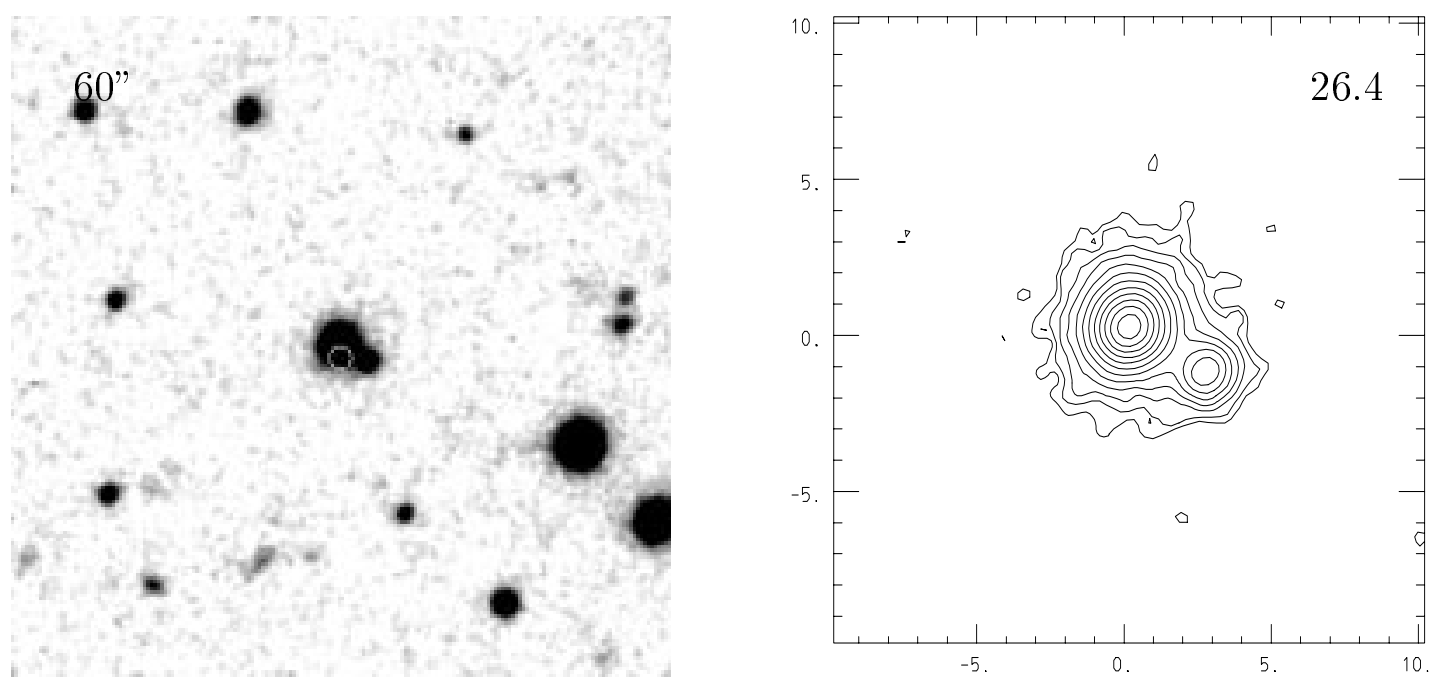

RC 1740+0502
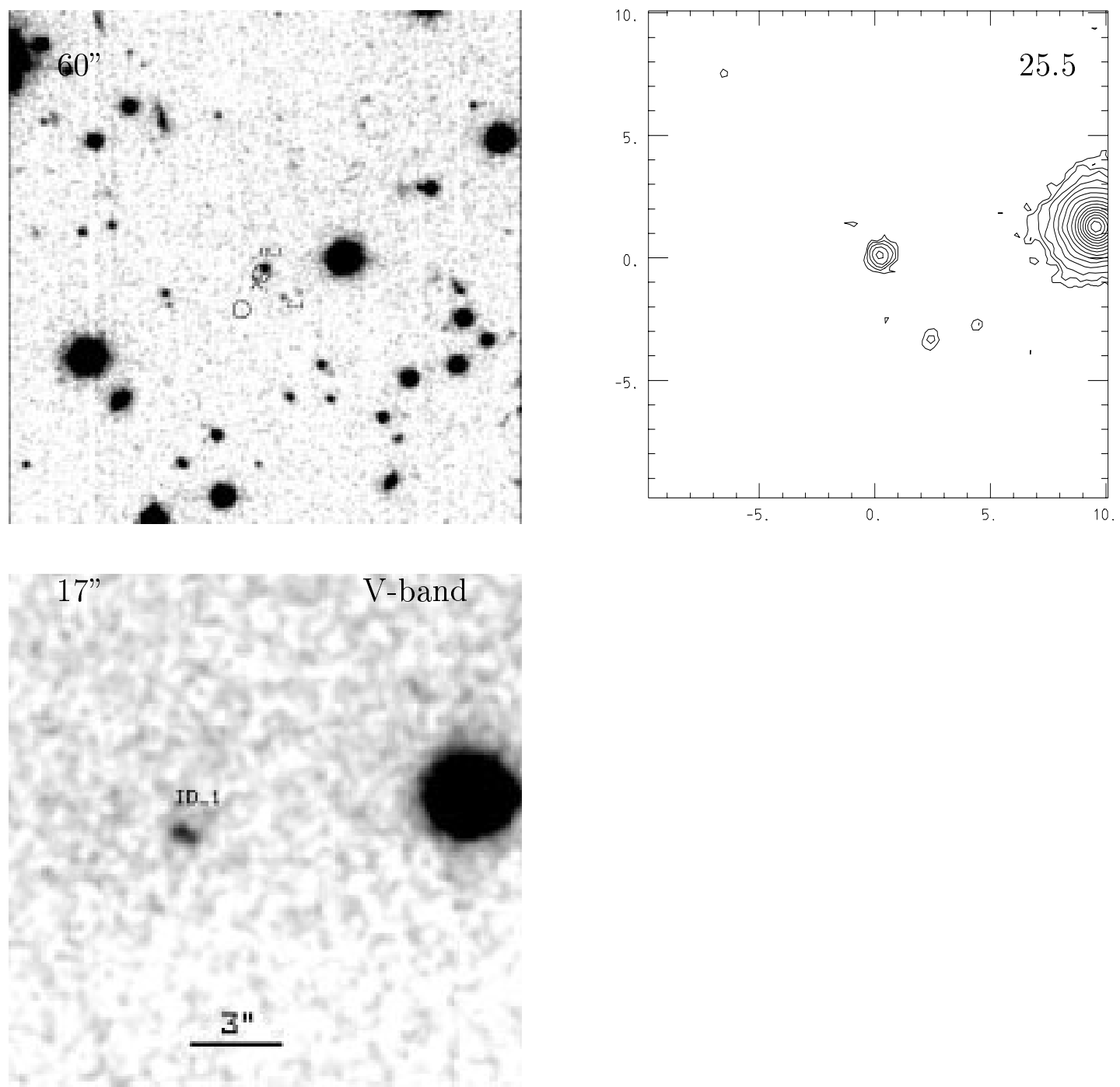

Fig. 3. continued 
$\mathrm{RC} 0459+0456$

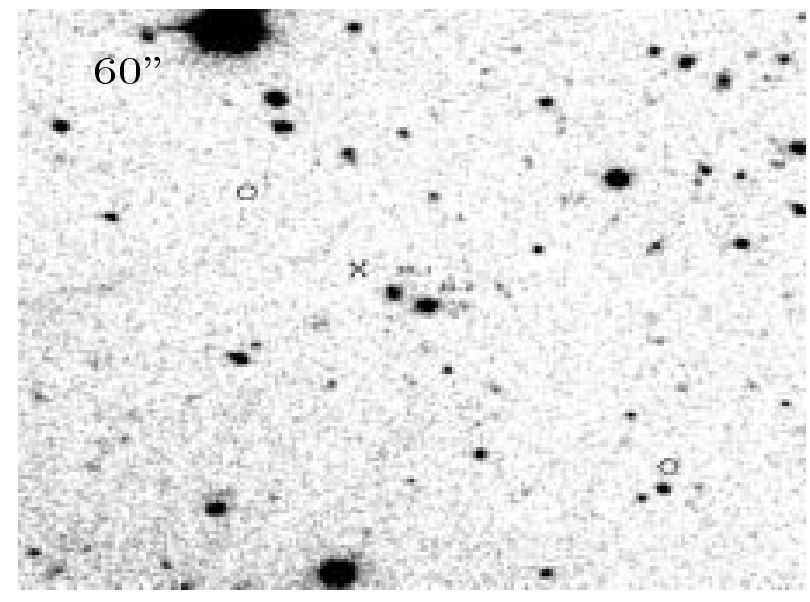

RC 1219+0446

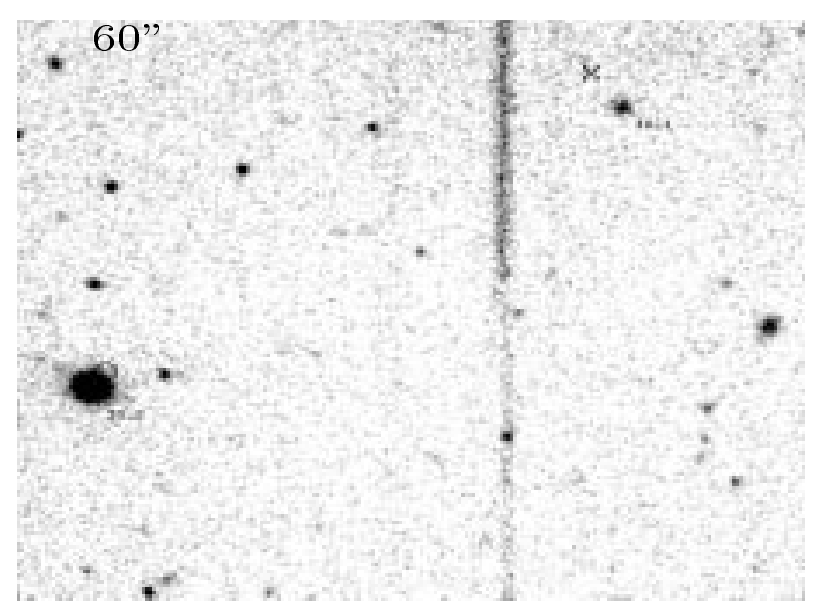

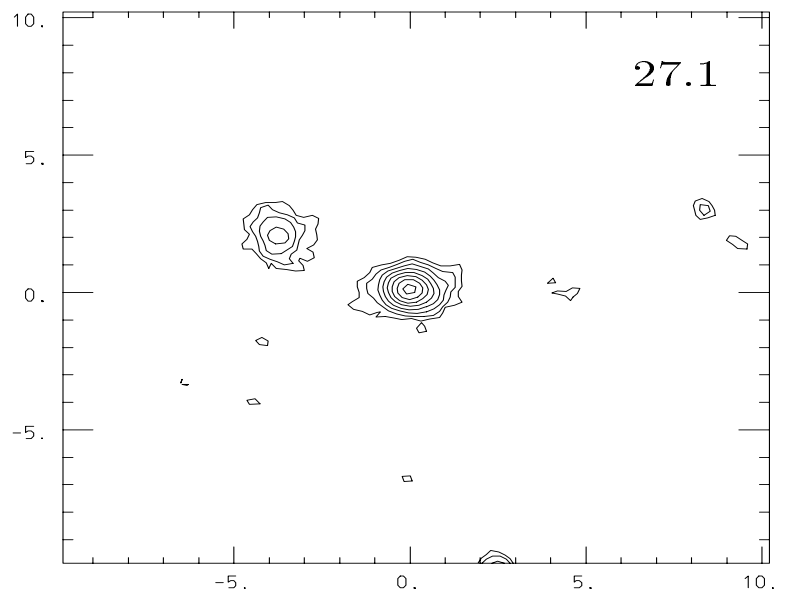

RC $1735+0454$

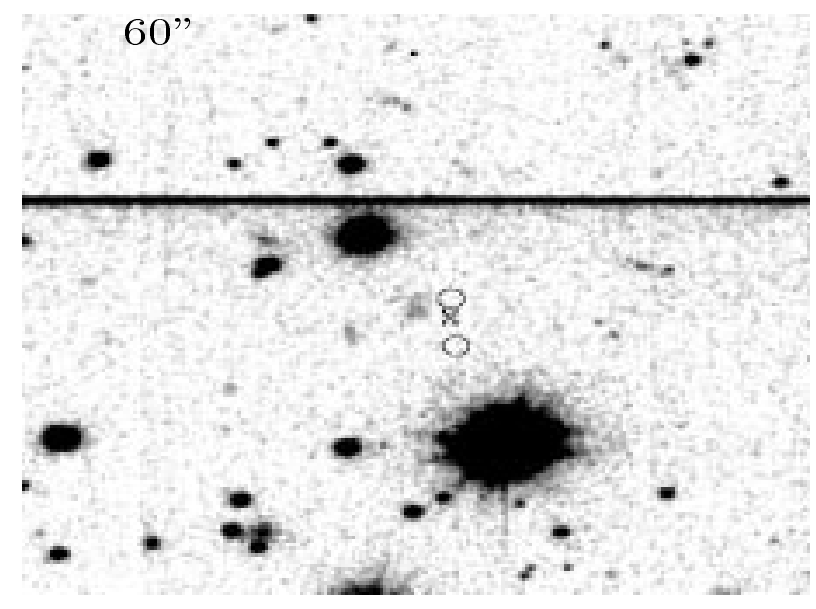

Fig. 4. Grey scale images of all the uncertain identifications (see Sect. 3.2). The images are produced in the same way as for Fig. 3. For RC $0459+0456$ we also give an $R$-band contour map

\subsection{Uncertain identifications}

\section{RC $0459+0456$}

(MRC 0456+048) This source has two candidates for optical identification in K95b. Id1 $\left(m_{R}=22.08\right)$ is an elongated galaxy with roughly the same position angle as the radio source. This object has a companion to the west $(\operatorname{Id} 2)$. This is a marginally resolved point-like source, hence it might be a quasar with a host galaxy $\left(m_{R}=21.12\right)$. FWHM of the id2 0 "' 66 compares with $F W H M$ of a field star 0 ". 62 .

\section{RC 1219+0446}

This is the largest radio source in our sample $\left(118^{\prime \prime}\right)$. The nature of the source remains unclear and it is possible that there are indeed two independent radio sources. If this is one source, then a possible identification would be a faint, rather round galaxy $(\operatorname{Id} 1) 5^{\prime \prime}$ from the centre of radio source $\left(m_{R}=21.9\right)$. On the other hand if the southern radio lobe is an independent object, the identification could be an unresolved object (Id2) $2^{\prime \prime}$ southeast from the radio source $\left(m_{R}=17.88\right)$.

\section{RC 1735+0454}

The possible optical counterpart is $\sim 3^{\prime \prime}$ to the east of the radio source. The galaxy has several components and it is elongated in a north south direction. The identification should be confirmed by future observations.

\subsection{Faint objects}

\section{RC $0743+0455$}

This object is very faint and hardly visible in the $30 \mathrm{~min}$ exposure.

\section{RC 1333+0451}

The radio source is compact. There is a faint extended emission exactly at the position of the radio source.

\section{RC 1609+0456}

New 6-m telescope measurements find an object with 


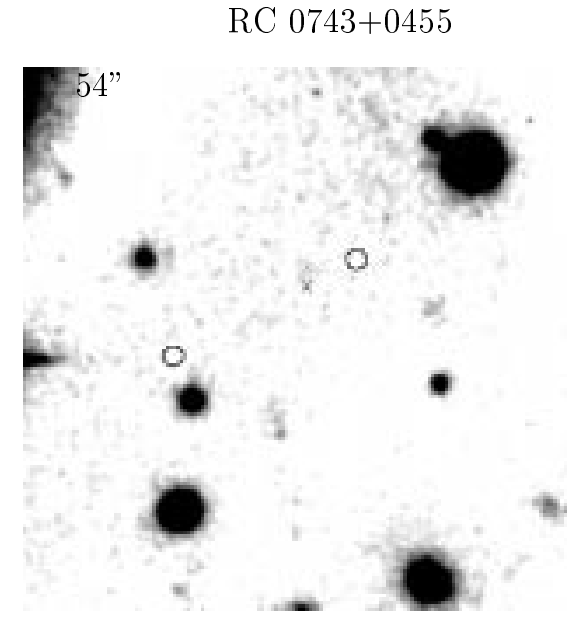

$\mathrm{RC} 1609+0456$

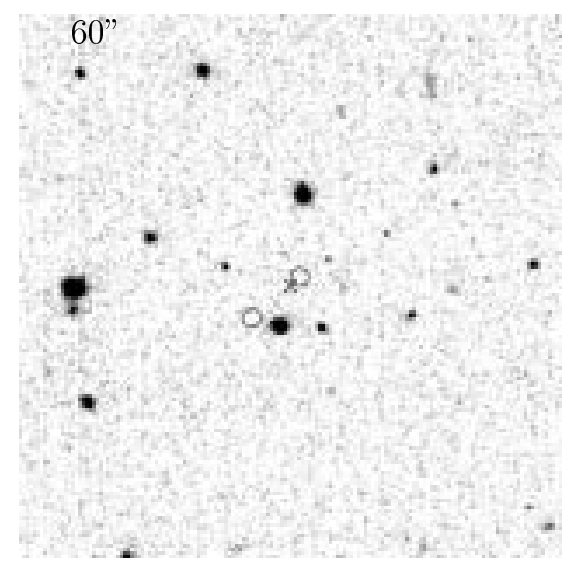

Fig. 5. Grey scale images of the faint objects (see Sect. 3.3)

$m_{R} \sim 25.5$ exactly at the position of the radio source. Our $600 \mathrm{~s}$ exposures are not deep enough to detect this object. The bright nearby object is unresolved and $B V R I$ photometry by K95b suggests it to be a star.

\section{Concluding remarks}

Excepting the quasar RC 2036+0451, the present galaxies do not have measured redshifts as yet. Hence, it is interesting to ask whether optical morphology provides information on the redshift distribution of the sample. Other properties, for example the Hubble diagram of the RC/USS sample, suggest (Sect. 1.3) that it contains galaxies with $z \gtrsim 0.7$.

Of the 22 observed objects, three are extremely faint and three others are either faint or have several possible identifications. Of the remaining 16 objects, 5 are unresolved. This is roughly the same fraction of point sources which Röttgering et al. (1995) found, but slightly more than $\mathrm{Lu}$ et al. (1996) found from their "distant" sample
$\mathrm{RC} 1333+0451$

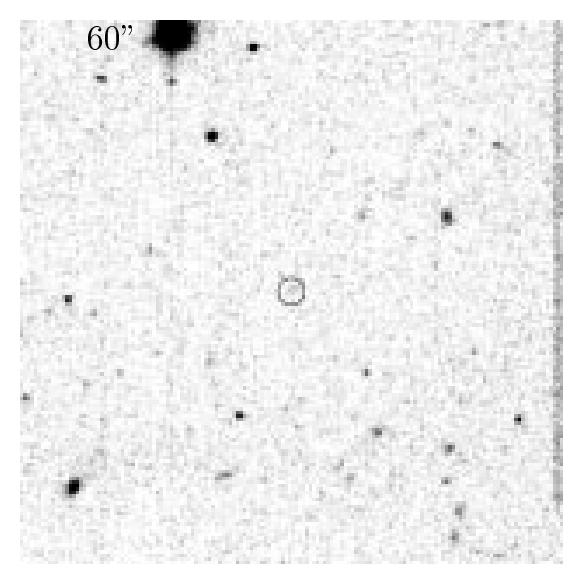

$\left(S_{1.4 \mathrm{GHz}}>35 \mathrm{mJy}\right)$, which does not have radio spectral index selection criteria. Typically RC/USS objects have a multicomponent structure with extended emission. This compares with HST images which have shown that about $30 \%$ of intermediate redshift $3 \mathrm{CR}$ galaxies have distorted morphology (De Koff et al. 1996). More distant $(z \sim 1)$ 3CR galaxies have typically multicomponent structure with diffuse extended emission (Best et al. 1996). The ellipticity (e) of the current sample (11 objects) ranges from 0.05 to 0.57 , with a mean of 0.25 . Taking into account the measurement errors these values agree with studies by Rigler et al. (1992) for 3C galaxies $(e=0.19)$ and by Röttgering et al. (1995) USS sample $(e=0.33)$.

Visual inspection of the images in Fig. 3 suggests that about half of the objects have a companion with comparable brightness within $10^{\prime \prime}$. We examined the excess of companion galaxies along the radio axis suggested by Röttgering et al. (1996). Our sample has 7 resolved objects with $3^{\prime \prime}<$ LAS $<20^{\prime \prime}$. Only two of these have companion galaxies (RC 1152+0449 and RC 1510+0438). 
RC $1152+0449$ has one companion almost exactly on the extension of the radio axis. RC $1510+0438$ has two companions, the brighter one being along the radio axis and the fainter one being perpendicular. It is also interesting to note that both of these "aligned" companions are about 1 magnitude fainter than the object. Four out of 16 objects have an apparent excess of companions and hence may be situated in a cluster of galaxies.

We can conclude that the morphology of the present weak radio flux USS population is close to that observed by e.g HST in high- $z$ radio galaxies. The results of this study make it imperative to measure spectroscopic redshifts for the RC/USS galaxies. Especially, the fainter flux limit makes it very interesting to see where in the Hubble diagram (both $R$ and $K$, the latter magnitudes still lacking) the RC-sources are found (cf. Eales et al. 1997). Naturally, the number of well observed RC/USS galaxies is still small. A more conclusive discussion of morphological features must wait until we complete the observations in the range $22^{\mathrm{h}}<\mathrm{RA}<4^{\mathrm{h}}$ for which $6 \mathrm{~m}$ telescope identifications are now available. Also, we intend to reobserve the cases of poor seeing in the present sample.

Acknowledgements. The authors thank the anonymous referee for useful comments. This work has been partially supported by grants from the Russian Foundation of Basic Research 9502-03783 and 96-02-16597 and the "Astronomy" programme project 2-296 and 1.2.1.2., 1.2.2.4. Yu.V.B. acknowledges the support of the Russian "Integration" project No. 578. TP acknowledges financial support from the Wihuri foundation. This work has been supported by the Academy of Finland (project Cosmology in the local galaxy Universe). This research has made use of the NASA/IPAC Extragalactic Database (NED) which is operated by the Jet Propulsion Laboratory, California Institute of Technology, under contract with the National Aeronautics and Space Administration. The National Radio Astronomy Observatory is a facility of the National Science Foundation operated under cooperative agreement by Associated Universities, Inc.

\section{References}

Allington-Smith J.R., Spinrad H., Djorgovski S., Liebert J., 1988, MNRAS 234, 1091

Best P.N., Longair M.S., Röttgering H.J.A., 1996, MNRAS 280, L9

Blumenthal G., Miley G., 1979, A\&A 80, 13

Carilli, Röttgering H.J.A., van Ojik R., Miley G.K., van Breugel W.J.M., 1997, ApJS 109, 1

Chambers K.C., Miley G.K., van Breugel W.J.M., 1988, ApJ 327, L47

de Breuck C., van Breugel W.J.M., Röttgering H.J.A., Miley G.K., 1997 astro-ph/9705056

De Koff S., Baum S.A., Sparks W.B., et al., 1996, ApJS 107, 621

Djorgovski S., Spinrad H., McCarthy P.J., et al., 1988, AJ 96, 836

Douglas J.N., Bash F.N., Bozyan F.A., Torrence G.W., Wolfe C., 1996, AJ 111, 1945
Dunlop J.S., Peackok J.A., 1993, MNRAS 263, 936

Eales S.A., Rawlings S., Dickinson M., et al., 1993, ApJ 409, 578

Eales S.A., Rawlings S., Law-Green D., Cotter G., Lacy M., 1997, MNRAS 291, 593

Fanaroff B.L., Riley J.M., 1974, MNRAS 167, 31

Fiorucci M., Tosti G., 1996, A\&AS 116, 403

Goss V.M., Parijskij Yu.N., Soboleva N.S., et al., 1992, AZh 69,673

Hammer F., Le Fevre O., 1990, ApJ 357, 38

Irwin M., Maddox S., McMahon R., 1994, Spectrum 2, 14

Kellermann K., Wall J., 1987, in: Observational Cosmology, Hewitt A., Burbidge G., Fang A.Z. (eds.), IAU Symp. 124, 545

Kopylov A.I., Goss V.M., Parijskij Yu.N., et al., 1995a, Astron. Rep. 39, 383

Kopylov A.I., Goss V.M., Parijskij Yu.N., et al., 1995b, Astron. Rep. 39, 543 (K95b)

Kristian J., Sandage A., Westphal J.A., 1978, ApJ 221, 383

Lacy M., Miley G., Rawlings S., et al., 1994, MNRAS 271, 504

Landolt A.U., 1992, AJ 104, 340

Lasker B.M., Sturch C.R., Mclean B.J., Russell J.L., Jenkner H., Shara M.M., 1990, AJ 99, 2019

LeFevre O., Hammer F., Nottale L., Mazure A., Christian C., 1988, ApJ 324, L1

LeFevre O., Hammer F., 1988, ApJ 333, L37

Lilly S.J., 1988, ApJ 333, 161

Lilly S.J., 1989, ApJ 340, 77

Lu N.Y., Hoffman G.L., Salpeter E.E., Houck J.R., 1996, ApJS 103,331

Maxfield L., Thompson D., Djorgovski S., Vigotti M., Grueff G., 1995, PASP 107, 369

McCarthy P.J., 1993, ARA\&A 31, 639

McCarthy P.J., Spinrad H., Djorgovski S., et al., 1987, ApJ 319, L39

McCarthy P.J., Kapahi V.K., van Breugel W., Subrahmanya C.R., 1990, AJ 100, 1014

McCarthy P.J., van Breugel W., Kapahi V.K., Subrahmanya C.R., 1991, AJ 102, 522

McCarthy P.J., Kapahi V.K., van Breugel W., et al., 1996, ApJS 107, 19

Miley G.K., Chambers K.C., van Breugel W.J.M., Macchetto F., 1992, ApJ 401, L69

Nilsson K., Valtonen M.J., Kotilainen J., Jaakkola T., 1993, ApJ 413, 453

Owen F.N., Keel W.C., 1995, AJ 109, 486

Parijskij Yu.N., Korolkov D., 1986, Ap. Space Phys. Rev. 5, 40

Parijskij Yu.N., Bursov N.N., Lipovka N.M., Soboleva N.S., Temirova A.V., 1991, A\&AS 87, 1

Parijskij Yu.N., Bursov N.N., Lipovka N.M., et al., 1992, A\&AS 96, 583

Parijskij Yu.N., Goss V.M., Kopylov A.I., et al., 1996a, Bull. SAO 40,5

Parijskij Yu.N., Soboleva N.S., Goss V.M., et al., 1996b, in: Extragalactic Radio Sources, Ekers R. et al. (eds.), IAU Symposium No. 175, Kluwer

Parijskij Yu.N., Kopylov A.I., Soboleva N.S., et al., 1997 in: International School of Astrophysics "D Chalonge" 7th Course, Current Topics In Astrofundamental Physics (in press)

Peacock J.A., 1997, MNRAS 284, 885 
Peacock J.A., Nicholson D., 1991, MNRAS 253, 307

Rigler M.A., Lilly S.J., Stockton A., Hammer F., Le Fevre O., 1992, ApJ 385, 61

Röttgering H.J.A., Lacy M., Miley G.K., Chambers, Saunders R., 1994, A\&AS 108, 79

Röttgering H.J.A., Miley G.K., Chambers K.C., Macchetto F., 1995, A\&AS 114, 51

Röttgering H.J.A., West M.J., Miley G.K., Chambers K.C., 1996, A\&A 307, 376

Soboleva N.S., Parijskij Yu.N., Naugol'naya M.N., 1994, Astron. Rep. 38, 605

Spinrad H., Dey A., Graham J.R., 1995, ApJ 438, L51
Thompson D., Djorgovsk S., Vigotti M., Grueff G., 1994, AJ 108,828

Tielens A., Miley G., Willis A., 1979, A\&AS 35, 153

Valdes F., Tyson J., Jarvis J.A., 1983, ApJ 271, 431

van Ojik R., Röttgering H.J.A., Miley G.K., Bremer M.N., Macchetto F., Chambers K.C., 1994, A\&A 289, 54

van Ojik R., Röttgering H.J.A., Miley G.K., Hunstead R.W., 1997, A\&A 317, 358

Veron-Cetty M.P., Veron P., 1996, ESO Scientific Report 17

Windhorst R.A., Burstein D., Mathis D.F., et al., 1991, ApJ 380,362 